\title{
Statistical framework for evaluation of climate model simulations by use of climate proxy data from the last millennium - Part 3: Practical considerations, relaxed assumptions, and using tree-ring data to address the amplitude of solar forcing
}

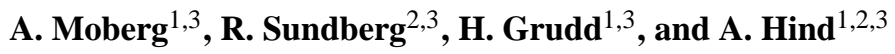 \\ ${ }^{1}$ Department of Physical Geography, Stockholm University, Stockholm, Sweden \\ ${ }^{2}$ Division of Mathematical Statistics, Department of Mathematics, Stockholm University, Stockholm, Sweden \\ ${ }^{3}$ Bolin Centre for Climate Research, Stockholm University, Stockholm, Sweden \\ Correspondence to: A. Moberg (anders.moberg@natgeo.su.se)
}

Received: 13 May 2014 - Published in Clim. Past Discuss.: 26 June 2014

Revised: 26 January 2015 - Accepted: 26 January 2015 - Published: 12 March 2015

\begin{abstract}
A statistical framework for evaluation of climate model simulations by comparison with climate observations from instrumental and proxy data (part 1 in this series) is improved by the relaxation of two assumptions. This allows autocorrelation in the statistical model for simulated internal climate variability and enables direct comparison of two alternative forced simulations to test whether one fits the observations significantly better than the other. The extended framework is applied to a set of simulations driven with forcings for the pre-industrial period 1000-1849 CE and 15 tree-ring-based temperature proxy series. Simulations run with only one external forcing (land use, volcanic, smallamplitude solar, or large-amplitude solar) do not significantly capture the variability in the tree-ring data - although the simulation with volcanic forcing does so for some experiment settings. When all forcings are combined (using either the small- or large-amplitude solar forcing), including also orbital, greenhouse-gas and non-volcanic aerosol forcing, and additionally used to produce small simulation ensembles starting from slightly different initial ocean conditions, the resulting simulations are highly capable of capturing some observed variability. Nevertheless, for some choices in the experiment design, they are not significantly closer to the observations than when unforced simulations are used, due to highly variable results between regions. It is also not possible to tell whether the small-amplitude or large-amplitude solar forcing causes the multiple-forcing simulations to be closer to the reconstructed temperature variability. Proxy data from
\end{abstract}

more regions and of more types, or representing larger regions and complementary seasons, are apparently needed for more conclusive results from model-data comparisons in the last millennium.

\section{Introduction}

While much of our knowledge about climate changes in the past emerges from evidence in various natural archives (Wanner et al., 2008; Jones et al., 2009), experiments with climate models help us to understand physical mechanisms behind the observed changes and may also help constrain projections of future climate changes (Schmidt, 2010). The last millennium - prior to the onset of the industrial era around $1850 \mathrm{CE}$ - provides an opportunity to test hypotheses about the role of external drivers, in particular orbital forcing, solar variability, volcanic aerosols, land use/land cover changes and variations in greenhouse gas levels, under climate conditions relatively close to those of today (Jungclaus et al., 2010; Schmidt et al., 2011; Landrum et al., 2012; Fernández-Donado et al., 2013; Sueyoshi et al., 2013). A constantly growing number of proxy-based reconstructions and model-based simulations of past climate variations implies an increasing need for statistical methods for comparing data of the two kinds. Examples of this are found in data assimilation (Goosse et al., 2012; Widmann et al., 2010), detection and attribution studies (Hegerl et al., 2007, 2011; Schurer et al., 2014), and es- 
timation of climate sensitivity (Hegerl et al., 2006). So far, the available methods cannot, however, account for the full complexity of the situation, e.g. the often time-varying quality and statistical precision of climate proxy data. It is also not clear how to determine the optimal spatial domain that a particular proxy record should represent in a model-data comparison. There is thus a need for more theoretical work in this context.

Based on theoretical considerations and some assumptions, Sundberg et al. (2012, henceforth SUN12) formulated a statistical framework for evaluation of climate model simulations, primarily for the last millennium. Their goal was to develop tools for an unbiased ranking of a set of alternative forced simulations in terms of their hypothetical distance to the unobservable true temperature history, while using noisy proxy records and instrumental observations as approximations to the true temperature variability. The alternative simulations in this context (in the rest of this paper) are obtained with one and the same climate model that has been run several times with alternative choices of the temporal evolution of external forcing conditions (e.g. alternative solar or volcanic forcing sequences). They may, however, also be different simulations obtained under alternative choices of parametrization of small-scale processes in one and the same climate model under exactly the same forcing conditions. In a companion pseudoproxy experiment, Hind et al. (2012) investigated the possibility of determining whether climate model simulations, driven by various external forcings, were able to explain past temperature variability in a situation where the "true" past temperature history, the forcing history and the proxy noise were known by design.

Here, we contribute further to the SUN12 work by discussing practical considerations arising when using real proxy data series that represent different seasons and regions of different size, having different lengths and statistical precision. To this end, we select a set of 15 tree-ring-based temperature reconstructions, spread across North America, Eurasia and Oceania, which we use together with the same set of global climate model simulations (Jungclaus et al., 2010) as used by Hind et al. (2012). Another goal is to present an extension of the SUN12 framework by relaxing two of its assumptions. This makes it possible, first, to allow some autocorrelation structures in the simulated temperatures and, second, to compare two alternative forced simulations directly to test whether one of them matches the observed climate variations significantly better than the other. SUN12 assumed no autocorrelation in the simulated internal (unforced) temperature variations and compared forced simulations only indirectly by testing whether each of them matched the observed climate variations better than a reference simulation with constant forcing. Although full details of the SUN12 framework are already provided in their original work, we summarize essential aspects here for the benefit of the reader. The extended framework is explained in detail in two appendices. Much of our discussion deals with practical issues which arise when applying the framework, for example concerning how to define geographical regions for model-data comparison, how to combine information representing different regions and seasons, and how to decide upon the time resolution to use in the analysis.

This work also serves as a companion study to the hemispheric-scale analysis by Hind and Moberg (2013), who attempted to determine which of two alternative solar forcing histories that, in the presence of other forcings, provided the best fit between simulated (Jungclaus et al., 2010) and reconstructed temperatures. The two solar forcing histories had either a 0.1 or $0.25 \%$ change in total solar irradiance since the Maunder Minimum period (i.e. 1645-1715 CE; c.f. Jungclaus et al., 2010; Lockwood, 2011; Schmidt et al., 2012; Fernández-Donado et al., 2013; Masson-Delmotte et al., 2013). As temperature proxies, Hind and Moberg (2013) used six hemispheric-scale temperature reconstructions: five based on multi-proxy compilations and one based solely on tree-ring data. They found, in most cases, a better match when the small-amplitude solar forcing was used, but results were not conclusive. This provokes questions regarding whether statistical model-data comparisons can tell which of the two alternative solar forcing histories is most correct. Tree-ring-based proxy data, which form the backbone of our knowledge of past temperature variations in the last millennium (Jones et al., 2009), have a potential to shed further light on this question. We apply the extended framework to the selected tree-ring data in an attempt to examine whether more conclusive results can be obtained. The current article is, however, mainly intended as a methodology study where the model-data analysis serves as a relevant demonstration case.

\section{Statistical framework}

To obtain a statistical methodology for ranking a set of plausible alternative forced simulations, and for identifying forced simulations fitting the observed temperature variations significantly better than an unforced model, SUN12 proposed a type of regional (or local) statistical model relating a climate model simulation time series $(x)$ via the (unobservable) true temperature sequence $(\tau)$ to the instrumental temperature measurements and temperature proxy data series for the region of interest. Instrumental measurements and proxy data (used only when the former are missing) are here jointly called "observations" and denoted $z$. For more explicit model formulations, see also Appendices A and B.

Section 4 of SUN12 demonstrated that, for unbiased ranking, the calibration of proxy data should aim at achieving the right scaling factor of the true temperature $(\tau)$ component in the proxy, with the noise component superimposed. Perfectly calibrated temperature proxy data (or instrumental data) could thus be written $z=\tau+\epsilon$, where $\epsilon$ is a measurement error type term (noise), uncorrelated with $\tau$. A remark 
in Sect. 4 of SUN12 discusses this type of calibration in light of recent literature (e.g. Hegerl et al., 2007; Ammann et al., 2010; Kutzbach et al., 2011; Moberg and Brattström, 2011) on the use of errors-in-variables models within a palaeoclimate reconstruction context. The methodology allows the variance of the noise term $\epsilon$ to vary with time, depending on how the precision of the observations varies. Thus, an entire $z$ sequence may be composed of different segments, each with its characteristic noise variance. Typically, one or more of the segments will consist of instrumental measurements, with noise variance generally expected to be smaller than in proxy segments.

Based on their statistical models, SUN12 developed two test statistics for comparing climate model simulation data with observations. First, before any attempt is made to rank alternative model simulations, testing should be done to establish whether a statistically significant positive correlation can be seen between a simulation series and the observations, because otherwise there is no evidence that the simulations and the true temperature share any effect of the forcing under study.

The correlation pre-test was formulated in SUN12 (Sect. 8) in terms of a regression type statistic, denoted $R(x, z)$. This formulation allowed weighting of the proxy data according to their presumed precision ${ }^{1}$ at the same time as it allowed conditioning on the observed climate as given and arbitrary. It is important to note, however, that high correlation does not mean that the forcing (or the response to it) is of the right size in the climate model simulation. In particular, a magnified forcing effect in $x$ necessarily increases the correlation, presuming that there is such a correlation.

Assuming next that a correlation has been established, a distance between a simulation sequence and an observation sequence is formed as a weighted mean squared distance, $D_{\mathrm{w}}^{2}$ :

$D_{\mathrm{w}}^{2}(x, z)=\frac{1}{n} \sum_{i=1}^{n} w_{i}\left(x_{i}-z_{i}\right)^{2}$.

Here, $n$ is the number of time steps in the sequence. It was argued in SUN12 that the weights $w_{i}$ should be chosen proportional to the inverses of the variances of $x_{i}-z_{i}$. The arbitrary proportionality constant was chosen to achieve $w_{i} \leq 1$, with equality for error-free observations of the true climate variable $\tau_{i}$. Through the weights $w_{i}$ the framework allows and adjusts for a temporally varying statistical precision of

\footnotetext{
${ }^{1}$ A principle for the choice of weights denoted $\widetilde{w}$ was proposed in SUN12. However, the explicit formulas given there (Sect. 8, p. 1348 , right column, first paragraph) did not satisfy this principle, but gave smaller weights to lower precision data than intended. The published formulas for $\widetilde{w}$ should instead be read as formulas for the squared weights, $\widetilde{w}^{2}$. In the present study, this makes almost no difference, because the weights are taken to be constant through time for each region, but it may have a stronger effect on data where weights are allowed to change with time.
}

the proxies. A time segment with low precision will receive a small weight $w_{i}$. For more details, see Sect. 5 of SUN12.

When an ensemble of simulations driven by the same forcing (but differing in their initial conditions) is available, they should all be used in an averaging process. This can be done in two different ways. Either a $D_{\mathrm{w}}^{2}$ value is computed for each simulation and the average of these values is used or, alternatively, the averaging is made of the simulation time sequences in the ensemble, before a $D_{\mathrm{w}}^{2}$ value is computed for this ensemble-mean time sequence. In SUN12, this was referred to as averaging "outside" and "inside", respectively, and was discussed primarily in their Sect. 6 and Appendix A. The theoretical discussion showed that the latter should be somewhat more precise, but with a possible bias. The pseudoproxy study by Hind et al. (2012) indicated that the inside method could be more effective, after bias correction. Later insight has told that the weights $w_{i}$ used in the inside method should rather be chosen differently such that the bias is eliminated. However, the theoretical gain of the inside method appears not to be very large, and the method has given more unstable results (not shown) than outside averaging. Hence, we are so far not in a position to recommend the inside method for testing, and, in any case, it should not be used for the different task of ranking models. In the present study we therefore only use outside averaging.

For comparison of different forced models, SUN12 used a normalized version of $D_{\mathrm{w}}^{2}$ rather than $D_{\mathrm{w}}^{2}$ itself. First, all $D_{\mathrm{w}}^{2}$ were replaced by their differences from the $D_{\mathrm{w}}^{2}$ of an unforced reference model (data $x^{*}$ ),

$T\left(x, x^{*}, z\right)=D_{\mathrm{w}}^{2}(x, z)-D_{\mathrm{w}}^{2}\left(x^{*}, z\right)$.

Thus, a (relatively large) negative value of $T\left(x, x^{*}, z\right)$ is needed to show that a forced model fits the observations better than the unforced reference. The question "how large?" is answered by scale-normalizing the $T\left(x, x^{*}, z\right)$ value by its standard error (square root of variance), calculated under the null hypothesis:

\section{$H_{0}$ : The forced climate model is equivalent to the unforced} reference model.

In Sect. 6 of SUN12, a formula is derived for the standard error of $T\left(x, x^{*}, z\right)$, depending only on the reference model output. The corresponding test statistic, formed by dividing $T$ by its standard error, can be used not only for testing the hypothesis $H_{0}$ but also for regional ranking of different individual forced simulations, or more generally for ranking different forced model ensembles when they all have equally many members (replicates). In the even more general case of forced model ensembles with unequally many replicates, the test statistic will be misleading for ranking, and instead the statistic $T$ itself should be used for ranking.

The test statistics for correlation and distance were derived under specific assumptions on the climate model simulations (whereas the true climate was arbitrary). For the purpose of 
model ranking only, this need not be considered a problem, but in their role as test statistics we want them to be robust against imperfections in the statistical model assumptions. In particular, we want to relax the following assumptions from SUN12:

- assumed lack of autocorrelation in the reference model simulations, i.e. these are statistically represented by white noise;

- truly unforced reference model, so in particular no joint time-varying forcing in $x$ and $x^{*}$.

Concerning the first assumption, it is well known that internal temperature variation can show autocorrelation, because the climate system acts as an integrator of the shortterm weather variations (Hasselmann, 1976). Depending on timescale (e.g. annual or decadal), short or long memory dominates. Vyushin et al. (2012) found that a first-order autoregressive representation $(\mathrm{AR}(1))$ and a power law can be seen as lower and upper bounds for characterizing this persistence. Hind et al. (2012) and Hind and Moberg (2013) attempted to avoid this problem by using quite long time units in their studies (30 and 20 years). This was empirically justified, as unforced temperatures in simulations they used were found to be compatible with white noise for these time units in relevant spatial and seasonal domains. Nevertheless, the knowledge that simulated unforced temperature variability can show autocorrelation motivates an extension of the SUN12 framework. Here we extend the theory by allowing unforced simulated temperatures to follow a short-memory time series model, in particular AR(1). It is shown in Appendix A how this is achieved with simple adjustment factors for the variances of the $R$ and $T$ statistics, including a discussion on how this is affected by the choice of time unit.

The second assumption must be relaxed in order to study the influence of two or more forcings added sequentially to a climate model, or to compare simulations with forcings of a similar type to see whether one fits significantly better than the other. Sequentially included forcings has been implemented, for example, by Phipps et al. (2013), but is not satisfied by the Jungclaus et al. (2010) set of simulations. However, we want to use this data set to compare simulations driven by low- or high-amplitude solar forcings, and we demonstrate in Appendix B that this can be done by a significance test allowing a particular forcing to have influence on the real climate. The method is easily described. We simply calculate the standard error of the T statistic as if both simulations were unforced. This will keep us on the safe side. The correlation test, on the other hand, must be changed, such that we compare the two $R$ statistics with each other and not with zero.

For the question how data from several regions and/or seasons (represented by index $j$ ) should be combined into a single statistic for ranking or testing, SUN12 (Sect. 7) proposed the use of a linear combination $c_{j} T_{j}$ of the corresponding
$T$ statistics, where the coefficients $c_{j}$ indicate in principle arbitrary weights to be given to the regions/seasons. For ranking, the quantity $c_{j} T_{j}$ itself should be used (although this was not explicitly mentioned in SUN12). For the test statistic, however, we need the standard error of $c_{j} T_{j}$. This requires an expression for the variance/covariance matrix of the set of $T_{j}$ statistics, based on the individual region/season standard errors and correlations of the $T$ statistic, Eq. (1), leading to the final test statistic $U_{T}$ for each climate model under consideration:

$U_{T}=\frac{\sum_{j} c_{j} T_{j}}{\sqrt{\operatorname{Var}\left(\sum_{j} c_{j} T_{j}\right)}}$.

The denominator is the standard error of the numerator, and the test statistic $U_{T}$ is approximately $N(0,1)$-distributed under the null hypothesis $H_{0}$ that the forcing introduced has no systematic effect on the fit of the model for any site.

If the forcing used in a simulation experiment is realistic, and if, additionally, its simulated climate response is realistic, we expect to see negative observed $T$ and $U_{T}$ values, but if the simulation exaggerates the forcing effect (either because the forcing has too large variation or because the climate model is too sensitive to the forcing), we might see systematically positive values. If a forced simulation produces a result that is indistinguishable from an unforced simulation (or a forced reference model, as in Appendix B), we would expect to see statistically insignificant $T$ and $U_{T}$ values, around zero. The correlation statistics $R(x, z)$ can be combined in the same way as the $T$ statistics into an aggregated correlation test value $U_{R}$ (see SUN12, Sect. 8).

Before the statistical framework can be applied, the time resolution (time unit) to use for the model-data comparison must be decided upon. For reasonably correct test $p$ values, it is essential to select a time unit that does not seriously violate an assumption that the simulated temperature for the reference model is $\operatorname{AR}(1)$. It is also necessary to select the size and shape of the area that a certain temperature ( $\tau$ and $x$ ) represents. If areas of very different sizes are combined in the calculation of $U_{R}$ and $U_{T}$, this may be a motivation to choose correspondingly different weights $c_{j}$. Different statistical precision of the proxy data series $(z)$, however, does not motivate choosing different weights $c_{j}$, because such differences are already accounted for by the weights $w$ used in $D_{\mathrm{w}}^{2}$ and $R$. Data from different regions need not represent the same season. Regions may overlap and it is even possible to include data from different seasons for one and the same region. Proxy series from different regions may have different lengths. SUN12 proposed to achieve this by letting the number of time steps, $n$, be the same for all regions. Regions with shorter proxy records than the full analysis period will thus have no terms contributing to their $D_{\mathrm{w}}^{2}$ sums in periods when they have no data. This would be the same as having a proxy $z$ with zero correlation to the true temperature $\tau$, and thus a weight $w_{i}=0$ before the actual proxy record starts. 
Evidently, several decisions need to be made when applying the framework in practice. Some of these issues will be discussed in Sect. 4, while Sect. 3 explains and discusses the choice of data sets.

\section{Data}

\subsection{Climate model data}

We follow Hind et al. (2012) and Hind and Moberg (2013) and use the simulations by Jungclaus et al. (2010) made with the Max Planck Institute Earth System Model (MPI-ESM) ${ }^{2}$. This comprises an atmospheric model run at $\mathrm{T} 31\left(3.75^{\circ}\right)$ resolution and an ocean model run at a horizontal resolution varying between 22 and $350 \mathrm{~km}$. The MPI-ESM includes an interactive carbon cycle model comprising an ocean biogeochemistry model and a land surface scheme.

Jungclaus et al. (2010) performed several simulations with forcing histories starting at $800 \mathrm{CE}$ and a 3000-year-long unforced control experiment with orbital conditions as of $800 \mathrm{CE}$ and constant pre-industrial greenhouse gas levels. Forced simulations of two kinds were made: one set with only a single forcing (either solar, volcanic, or land cover change) and another set with multiple forcings (combining solar, volcanic and land cover with orbital and greenhouse gas forcing as well as with non-volcanic aerosols). Two alternative solar forcing histories were used: the small-amplitude one by Krivova et al. (2007) with a $0.1 \%$ change in total solar irradiance between the Maunder Minimum and the present, and the large-amplitude one by Bard et al. (2000) with a $0.25 \%$ change. These two solar forcing series are, however, not simply two versions of the same basic time series with a different scaling. The multiple-forcing simulations are available as two small ensembles, where individual members start from different ocean initial conditions at $800 \mathrm{CE}$. The "E1" ensemble, using the small-amplitude solar forcing, has five members, while the "E2" ensemble, using the large-amplitude solar forcing, has three members. For simplicity, we will often denote these two multiple-forced ensembles the "low" and "high" solar ensembles in the rest of this paper. Like Hind et al. (2012) and Hind and Moberg (2013), we use forced simulations $(x)$ from year 1000 CE onwards and split the control simulation into three 1000-yearlong segments to obtain a small ensemble of unforced simulations $\left(x^{*}\right)$ of the same length. We refer to Figs. 1 and 2 in Hind et al. (2012) for time series plots of all forcings and of simulated global mean land-only temperatures for the various simulations by Jungclaus et al. (2010).

\footnotetext{
${ }^{2}$ http://www.ncdc.noaa.gov//paleo/metadata/ noaa-model-10477.html
}

\subsection{Instrumental data}

Instrumental temperature data are needed for two purposes. First, SUN12 argued for using best possible data to maximize the statistical precision of the model-data comparison. Thus, in most cases, instrumental data should be used rather than proxy data within time periods when both exist. Second, instrumental data are needed to calibrate the proxy data. There are, however, several alternative temperature data sets to choose between (e.g. Brohan et al., 2006; Smith et al., 2008; Hansen et al., 2010; Morice et al., 2012).

Hind and Moberg (2013) used the CRUTEM3 land-only data set by Brohan et al. (2006), which has a $5^{\circ}$ resolution going back to 1850 . This data set is provided with estimates of the error term (due to various types of station errors, spatial sampling errors and systematic bias errors) in grid-box or larger-scale mean temperatures. However, Hind and Moberg (2013) could only incorporate these estimates in the SUN12 framework with assistance from the main author of Brohan et al. (2006), as error terms were not published for arbitrary regions and seasons. The updated land-plus-marine data set HadCRUT4 (Morice et al., 2012) is provided with more comprehensive quantitative information about various types of errors, partly dealt with by presenting grid-point temperatures as 100 slightly different ensemble members. This should make it possible to estimate relevant noise terms, although at the expense of extra programming. We have not tried this option here.

For the current study, we instead selected the GISS1200 gridded global temperature data set (Hansen et al., 2010), which goes back to 1880 . This data set uses a rather large search radius $(1200 \mathrm{~km})$ for averaging data from temperature stations in the calculation of each grid-point value. Therefore, GISS 1200 data are spatially and temporally rather complete in remote areas such as the North American and Eurasian subarctic regions, where several tree-ring chronologies are located but where few temperature stations - often with rather short records - are found. Despite its coarse spatial smoothing, GISS1200 is published at a rather fine grid $\left(2^{\circ}\right)$. This gives some flexibility when defining regions for temperature averages against which the tree-ring records are calibrated. Because the model and instrumental grids are different, we re-gridded the model grid to the same as for GISS1200 using bilinear interpolation to enable comparison of analogous regions. A drawback with using GISS1200 is that explicit information about the instrumental error term is not available. We have therefore simply subjectively assumed that the noise term always accounts for $5 \%$ of the total variance in instrumental temperature data, regardless of season and size of region. This is a limitation, but we checked the sensitivity of our results to the instrumental noise assumption by trying also 0,10 and $20 \%$. This had only a marginal effect (not shown) and did not affect any conclusions. 


\subsection{Tree-ring data}

Tree-ring data are available from many parts of the globe. They can be sensitive to climate in different seasons but always have annual resolution and often explain a substantial fraction of observed temperature or precipitation variations (Fritts, 1976; Hughes, 2002; Briffa et al., 2004; Hughes et al., 2011; St. George and Ault, 2014). Tree-ring data, from either ring width (TRW) or maximum density (MXD), are also the most extensively used proxies in temperature reconstructions for the last millennium (Jones et al., 2009). Here, we select 15 tree-ring records that start before $1500 \mathrm{CE}$ and which have been demonstrated to show a signal of temperature variability for a certain seasonal window. Four records are from North America, five from Europe, four from Asia and two from Oceania. Nine records start before $1000 \mathrm{CE}$ (i.e. they extend back to the start of our analysis period). Table 1 lists all records with their short names used here, data type (TRW or MXD), seasonal targets, first year used in analysis and references to literature that describes the records. Table 2 provides web links to data source files. One of the 15 records (from Cook et al., 2013) is a reconstruction of largescale temperatures derived from trees growing at a range of sites across eastern Asia, but the other 14 records are derived from trees growing at either single locations or rather small regions. We regard our selection as sufficiently complete for the purpose of this study, although there are, admittedly, other records that could potentially have also been included. It is not a problem that the Southern Hemisphere and Northern Hemisphere seasons are offset by half a year, because each site contributes its own $R$ and $T$ value to the $U$ statistics (see Sect. 2).

Twelve of the 15 tree-ring records have been developed using the regional curve standardization (RCS) technique (cf. Briffa et al., 1992), which can preserve variations on timescales longer than the life length of individual trees. This is essential here, as we are interested in studying long-term temperature variations, in particular to distinguish between small- and large-amplitude solar forcing simulations. "Individual standardization" (IND) will inevitably inhibit variations on longer timescales, as has been frequently discussed as the "segment length curse" problem in dendroclimatology (e.g. Cook et al., 1995). In fact, all standardization methods, whether applied as IND or RCS, will effectively remove a portion of the climate signal from the raw tree-ring data. Melvin and Briffa (2008) introduced a method that allows the simultaneous estimation of the tree-ring standardization curves and the common environmental signal that is embedded in the same tree-ring records within a region. This socalled "signal-free" (SF) iterative standardization method removes the influence of the common environmental (assumed climate) signal on the standardization curve, which reduces the trend distortion that can occur near the ends of a traditionally standardized chronology. The method can be applied on both IND and RCS standardization (Melvin and Briffa,
2014), but very few records have been created with this rather new technique. Three records in our collection were developed using SF in combination with RCS.

One additional comment should be made in context of the SUN12 framework. The number of trees used in a tree-ring chronology will most often vary through time; typically there are fewer trees in the earliest part of a chronology, but the sample size can vary very irregularly with time. These variations in sample size are known to cause temporal variations in the variance of a chronology. Osborn et al. (1997) proposed to adjust the chronology variance such that it is approximately the same at each time point as if, hypothetically, an infinite number of trees from within the actual region had been used. This type of variance adjustment is nowadays a standard procedure in dendroclimatology, and several records in our selection are processed this way. A somewhat similar variance adjustment is sometimes also applied to account for a varying number of chronologies used to build a composite temperature reconstruction, as, for example, in the records of Wilson et al. (2007) and Cook et al. (2013) used here. It may be that these variance adjustments induce a violation to a crucial assumption in the SUN12 framework, namely that a proxy sequence $z$ should be calibrated such that the true temperature component $\tau$ always has its correct variance, with the noise term $\epsilon$ superimposed. Undoing these adjustments is generally not possible without information that is only available to the original investigator, and it is beyond the scope of this study to attempt doing this. We merely point out this issue as a potential problem and simply regard published chronologies or temperature reconstructions as uncalibrated proxy sequences, which can be re-calibrated back to the start of the analysis period by using the statistical relationship to selected instrumental temperature data in a chosen calibration period.

\section{Practical considerations}

\subsection{Selecting seasons}

The first decision is to select the season that each proxy record will represent in the model-data comparison. As each original author team has generally spent considerable efforts on determining the most appropriate season for each record and as the SUN12 framework admits using all possible combinations of seasons - it seems most natural to follow the respective original judgements (see Table 1).

\subsection{Choosing calibration periods}

A time period (or time periods) is required for calibration of tree-ring data and, as we argue below (Sect. 4.3), for analysing the spatial pattern of correlations between tree-ring data and the instrumental temperature field. Our general recommendation is to use the longest meaningful calibration period for each record, and avoid using calibration data that are 
Table 1. Tree-ring temperature reconstructions used in this study, with seasonal representation as determined by the respective investigators. TRW - tree-ring width; MXD - maximum density; IND - individual standardization; RCS - regional curve standardization; SF - signal-free standardization. Short names and start year used in this study are also given.

\begin{tabular}{|c|c|c|c|c|c|c|}
\hline Name & Abbr. & Proxy & Stand. & Season & Start & Reference \\
\hline Gulf of Alaska & GOA & TRW & IND & Jan-Sep & 1000 & Wilson et al. (2007) \\
\hline Firth River & FIRTH & MXD & $\mathrm{RCS}+\mathrm{SF}$ & Jul-Aug & 1073 & Anchukaitis et al. (2013) \\
\hline Coppermine/Thelon ${ }^{\mathrm{a}}$ & $\mathrm{CT}$ & MXD & IND & May-Aug & 1492 & D'Arrigo et al. (2009) \\
\hline Canadian Rockies & CANR & MXD & $\mathrm{RCS}$ & May-Aug & 1000 & Luckman and Wilson (2005) \\
\hline Torneträsk & TORN & MXD & $\mathrm{RCS}+\mathrm{SF}$ & May-Aug & 1000 & Melvin et al. (2013) \\
\hline Jämtland & JAMT & MXD & $\mathrm{RCS}$ & Apr-Sep & 1107 & Gunnarson et al. (2011) \\
\hline Tatra & TATRA & TRW & RCS & May-Jun & 1040 & Büntgen et al. (2013) \\
\hline Alps & ALPS & MXD & RCS & Jun-Sep & 1000 & Büntgen et al. (2006) \\
\hline Pyrenees & PYR & MXD & $\mathrm{RCS}$ & May-Sep & 1260 & Dorado Liñán et al. (2012) \\
\hline Yamalia Combined & YAMC & $\mathrm{MXD}+\mathrm{TRW}$ & $\mathrm{RCS}+\mathrm{SF}$ & Jun-Jul & 1000 & Briffa et al. (2013) \\
\hline Avam-Taimyr & AVAMT & TRW & $\mathrm{RCS}$ & Jul & 1000 & Briffa et al. (2008) \\
\hline Yakutia $^{\mathrm{b}}$ & YAK & TRW & $\mathrm{RCS}$ & Jun-Jul & 1342 & D’Arrigo et al. (2006) \\
\hline East Asia ${ }^{c}$ & ASIA2k & TRW & other & Jun-Aug & 1000 & Cook et al. (2013) \\
\hline Tasmania & TASM & TRW & RCS & Nov-Apr & 1000 & Cook et al. (2000) \\
\hline New Zealand & $\mathrm{NZ}$ & TRW & RCS & Jan-Mar & 1000 & Cook et al. $(2002,2006)$ \\
\hline
\end{tabular}

${ }^{a}$ Arithmetic average of normalized Coppermine and Thelon data.

${ }^{\mathrm{b}}$ Seasonal representation as in Wilson (2004).

${ }^{c}$ Detailed information on standardization is not provided in Cook et al. (2013) but included a partial use of SF.

Table 2. Data sources for the tree-ring records.

\begin{tabular}{ll}
\hline Record & Source \\
\hline GOA & ftp://ftp.ncdc.noaa.gov/pub/data/paleo/treering/reconstructions/gulf_of_alaska/goa2007temp.txt \\
FIRTH & ftp://ftp.ncdc.noaa.gov/pub/data/paleo/treering/reconstructions/northamerica/usa/alaska/firth2013temperature.txt \\
CT & ftt://ftp.ncdc.noaa.gov/pub/data/paleo/treering/reconstructions/northamerica/usa/alaska/firth2013temperature.txt \\
CANR & ftp://ftp.ncdc.noaa.gov/pub/data/paleo/treering/reconstructions/canada/icefields-summer-maxt.txt \\
TORN & http://www.cru.uea.ac.uk/cru/papers/melvin2012holocene/TornFigs.zip \\
JAMT & ftp://ftp.ncdc.noaa.gov/pub/data/paleo/treering/reconstructions/europe/sweden/gunnarson2011temp.txt \\
TATRA & ftp://ftp.ncdc.noaa.gov/pub/data/paleo/treering/reconstructions/europe/tatra2013temp.txt \\
ALPS & ftp://ftp.ncdc.noaa.gov/pub/data/paleo/treering/reconstructions/europe/buentgen2011europe.txt \\
PYR & ftp://ftp.ncdc.noaa.gov/pub/data/paleo/pages2k/DatabaseS1-All-proxy-records.xlsx \\
YAMC & ftp://ftp.ncdc.noaa.gov/pub/data/paleo/treering/reconstructions/asia/russia/yamalia2013temp1000yr.txt \\
AVAMT & http://www.cru.uea.ac.uk/cru/papers/briffa2008philtrans/Column.prn \\
YAK & ftp://ftp.ncdc.noaa.gov/pub/data/paleo/treering/reconstructions/n_hem_temp/nhtemp-darrigo2006.txt \\
ASIA2k & ftp://ftp.ncdc.noaa.gov/pub/data/paleo/pages2k/DatabaseS2-Regional-Temperature-Reconstructions.xlsx \\
TASM & ftp://ftp.ncdc.noaa.gov/pub/data/paleo/treering/reconstructions/tasmania/tasmania_recon.txt \\
NZ & ftp://ftp.ncdc.noaa.gov/pub/data/paleo/pages2k/DatabaseS1-All-proxy-records.xlsx \\
\hline
\end{tabular}

known to be unrepresentative. For the current study, however, we take a simple pragmatic approach and use the same calibration periods as were used by each original investigator (see Table 3).

\subsection{Defining regions}

Defining the region that each tree-ring series will represent is a more challenging task. SUN12 stated (in their Sect. 2) that "typically, this region consists of a single grid box, but averages over several grid boxes can also be considered". A single grid-box temperature may perhaps maximize the statis- tical precision for calibration of a single tree-ring chronology, but climate model errors are typically largest at the gridbox scale and decrease with increasing spatial smoothing. It has therefore been recommended that some spatial averaging is applied when climate models are evaluated (Masson and Knutti, 2011). Also, one of our tree-ring records (ASIA2k) is derived from trees that grew in an area that extends over several grid boxes. Moreover, unforced temperature variability will have a larger influence in a single grid box as compared to an average of several grid boxes, where the forced part of the temperature variation will be more easily detected (e.g. Servonnat et al., 2010). Thus, as we are here primarily inter- 
Table 3. Selected information for each region: latitude/longitude boundaries, proxy data calibration period, and correlation $r$ with instrumental target, area weight $c_{j}$, and cluster weight $c_{j}$. The area weights are defined as the fraction of the global area. The cluster weights are explained in the text.

\begin{tabular}{lcccccc}
\hline Name & Lat. & Long. & Cal. period & $r$ & Area $c_{j}$ & Cluster $c_{j}$ \\
\hline GOA & $59-63^{\circ} \mathrm{N}$ & $135-161^{\circ} \mathrm{W}$ & $1899-1985$ & 0.55 & 0.12 & $1 / 4$ \\
FIRTH & $67-71^{\circ} \mathrm{N}$ & $125-149^{\circ} \mathrm{W}$ & $1897-2002$ & 0.63 & 0.08 & $1 / 4$ \\
CT & $61-71^{\circ} \mathrm{N}$ & $93-121^{\circ} \mathrm{W}$ & $1950-1979$ & 0.75 & 0.28 & $1 / 4$ \\
CANR & $47-59^{\circ} \mathrm{N}$ & $109-125^{\circ} \mathrm{W}$ & $1895-1994$ & 0.62 & 0.28 & $1 / 4$ \\
TORN & $63-71^{\circ} \mathrm{N}$ & $11-25^{\circ} \mathrm{E}$ & $1880-2006$ & 0.78 & 0.11 & $1 / 2$ \\
JAMT $^{\mathrm{a}}$ & $59-67^{\circ} \mathrm{N}$ & $7-27^{\circ} \mathrm{E}$ & $1880-2007$ & 0.75 & 0.18 & $1 / 2$ \\
TATRA & $45-53^{\circ} \mathrm{N}$ & $13-29^{\circ} \mathrm{E}$ & $1901-2009$ & 0.42 & 0.20 & $1 / 3$ \\
ALPS & $45-47^{\circ} \mathrm{N}$ & $3^{\circ} \mathrm{W}-11^{\circ} \mathrm{E}$ & $1911-2003$ & 0.72 & 0.03 & $1 / 3$ \\
PYR & $39-45^{\circ} \mathrm{N}$ & $7^{\circ} \mathrm{W}-5^{\circ} \mathrm{E}$ & $1900-2005$ & 0.64 & 0.13 & $1 / 3$ \\
YAMC & $61-75^{\circ} \mathrm{N}$ & $53-81^{\circ} \mathrm{E}$ & $1883-2005$ & 0.79 & 0.36 & $1 / 2$ \\
AVAMT & $65-75^{\circ} \mathrm{N}$ & $81-107^{\circ} \mathrm{E}$ & $1950-1994$ & 0.66 & 0.22 & $1 / 2$ \\
YAK & $65-73^{\circ} \mathrm{N}$ & $137-161^{\circ} \mathrm{E}$ & $1951-1980$ & 0.70 & 0.17 & 1 \\
ASIA2k & $37-55^{\circ} \mathrm{N}$ & $73-143^{\circ} \mathrm{E}$ & $1951-1989$ & 0.59 & 2.11 & 1 \\
TASM & $39-49^{\circ} \mathrm{S}$ & $127-159^{\circ} \mathrm{E}$ & $1886-1991$ & 0.52 & 0.56 & $1 / 2$ \\
NZ & $39-47^{\circ} \mathrm{S}$ & $163-177^{\circ} \mathrm{E}$ & $1894-1957$ & 0.45 & 0.20 & $1 / 2$ \\
\hline
\end{tabular}

${ }^{\mathrm{a}}$ Gunnarson et al. (2011) used 1870-2007, but GISS1200 starts in 1880.

b Calibration period as in Wilson (2004).

ested in seeing how well the model simulates the externally forced temperature variation, it appears recommendable to select an area that is large enough to detect the forced simulated temperature response but small enough that the actual proxy record provides a meaningful approximation of the true temperature variability.

Although we cannot give any precise recommendation on how to determine the optimal spatial domain that a proxy record should represent, we assume that there is also a similar need in data assimilation and detection and attribution studies. This appears to be an issue where more research is needed. At this stage, we can at least suggest a practically affordable way to semi-subjectively define a reasonable region for each tree-ring record. To this end, we plot and visually interpret the spatial field of correlations between each tree-ring record and the appropriate seasonal mean temperatures in GISS1200 data (Fig. 1). This correlation analysis is made using first-differenced data to minimize possibly spurious correlations due to both linear and nonlinear trends that do not reflect a direct physiological association between the temperatures in each growth season and the tree-ring data. This idea is similar to that adopted by Cook et al. (2013) in their screening to determine which individual tree-ring chronologies were positively correlated with grid-point temperatures, although they fitted an AR(1) model and removed this component from the data before calculating correlations. More generally, an often used class of models with nonstationarity is the ARIMA class of models, and for such models linear trend and nonstationarity are simultaneously eliminated by first forming a new series of first (or higher order) differences (Box et al., 2007, Ch. 4). In fact, Fritts (1976, p. 329) already proposed to use first differences in tree-ring research to study (by means of a sign test) whether or not sufficient similarity exists between actual and estimated climate data. We also experimented with a linear detrending of the data before performing the correlation analysis, but we found that the form and size of the regions where correlations are strong in this case does not depend crucially on the choice of first differences versus linear detrending method.

The spatial correlation analysis was undertaken for calibration periods chosen above. Each map was then visually inspected to determine an appropriate region. We did not attempt to define any objective criterion, but we combined information about (i) where correlations are strongest, (ii) where chronologies are located and (iii) information from the literature regarding which regions the data represent. For example, the TASM area is allowed to extend over much of the ocean surrounding Tasmania, because Cook et al. (2000) suggested their record as a proxy for large-scale sea surface temperature anomalies. As another example, we followed the observation by Cook et al. (2013) that the ASIA2k record best represents regional temperatures north of $36^{\circ} \mathrm{N}$. An additional constraint was the spatial resolution of the instrumental temperature grid $\left(2^{\circ}\right)$ and an account for the land/sea mask in the climate model and how this relates to the real land/sea borders. We did not attempt to merge all these pieces of information objectively, but our approach is merely an "expert judgement". The resulting regional representation for each tree-ring record is illustrated in Fig. 2 and the regional latitude/longitude boundaries as well as corresponding fractions of the global area are provided in Table 3. Together, the 15 regions represent $5 \%$ of the global area but their sizes differ remarkably. The largest region (ASIA2k) is 70 times 
larger than the smallest (ALPS) and alone comprises more than $40 \%$ of the total area of all regions put together.

\subsection{Selecting weights $c_{j}$}

The vastly different sizes of regions, as well as their uneven geographical distribution and different seasonal representation, motivates some suitable weights $c_{j}$ (explained in Sect. 2) being chosen. The simplest choice is to let all $c_{j}$ be equal, i.e. to regard all selected proxy records as equally important. Another intuitive choice is to use the area of each region as weight. A third alternative is to choose weights according to how much "new" or "additional" climate information each region contributes in comparison with the other regions. A fourth alternative could be to weight the regions by how easy it is to detect the externally forced variability. This alternative could be similar to optimization in detection and attribution studies (see e.g. Allen and Tett, 1999). Here, we try the first three alternatives and compare the results to see how sensitive $U_{R}$ and $U_{T}$ measures are to the choice of weights $c_{j}$. Moreover, we study the effect of excluding the three tree-ring records that were not RCS-standardized (GOA, CT, ASIA2k) and weight the remaining 12 regions equally.

The equal and area weights are straightforward. The latter are provided in Table 3. Note that the sum of $c_{j}$ need not be 1 . For the third alternative, we try a cluster analysis approach. This, however, requires some subjective decisions: one needs to choose a distance metric, a linkage method and also decide how many clusters to use. One also needs to decide which data to analyse. The full 3000-year control simulation is an adequate choice that provides a large sample representing unforced (internal) simulated climate variability. The quantity $1-r$, where $r$ is the sample correlation between regions, appears intuitively meaningful as a distance metric. Fig. 3 shows the result of a cluster analysis using nearest-neighbour linkage (Matlab, 2008). By choosing seven clusters, we obtain a geographically and climatologically meaningful grouping of regions: northern Scandinavia (JAMT, TORN), continental Europe (PYR, ALPS, TATRA), eastern Asia (ASIA2k), Oceania (TASM, NZ), northwestern Siberia (AVAMT, YAMC), northwestern North America (GOA, FIRTH, CT, CANR) and northeastern Siberia (YAK). We set the weights $c_{j}$ such that each cluster contributes oneseventh to the total. Within each cluster, the contributing regions are equally weighted. This gives cluster-based weights as listed in Table 3.

\subsection{Calibration of the tree-ring records}

The tree-ring data need to be re-calibrated to appropriate regional and seasonal mean temperatures. Thus, the GISS1200 seasonal mean temperatures are averaged within each region and calibration is made for the chosen calibration periods, following procedures explained in Sect. 4 of SUN12 under the assumption that instrumental noise variance accounts for $5 \%$ of the total observed temperature variance in each region (see Sect. 3.2). Moreover, as explained in Sect. 3.3, we assume that the statistical precision of each tree-ring record in the calibration period is also representative back to the start of the record. Table 3 lists correlations between each treering record and the corresponding instrumental temperature record, ranging from 0.42 to 0.79 . These correlations provide the information on the statistical precision of proxies that is used when calculating weights $w$. For each region, the calibrated tree-ring data sequence is then taken as the $z$ sequence to compare with the corresponding model sequence $x$. The variance contribution from the calibration uncertainty has not been considered in our analysis.

\subsection{Selecting analysis period}

Another decision concerns the time window for which $U_{R}$ and $U_{T}$ measures are computed. With our choice of data, the longest possible window would be 1000-2000 CE, which includes both pre-industrial conditions and the increasingly anthropogenically influenced industrial period. Our focus, however, is on natural forcings, which motivates exclusion of the industrial period. We choose to analyse the period 1000$1849 \mathrm{CE}$ to make it possible to directly compare our results with those from Hind and Moberg (2013). Thus, $z$ sequences in our model-data comparison do not include any instrumental data. If we had chosen to include data after 1880 , we would have used GISS data after 1880 and re-calibrated treering data before 1880 (see Sect. 2).

\subsection{Selecting time unit}

Finally, a time unit must be selected, i.e. the length of time periods over which we average temperatures to obtain the pairs of simulation $\left(x_{i}\right)$ and observation $\left(z_{i}\right)$ values to be compared. It should be noted, though, that the precise choice of time unit is not crucial. Empirically, this can be seen in Fig. 6. We must compromise between arguments for longer and shorter units of time, a matter regarded as a question of principle. Arguments for long units are a reduced autocorrelation in the reference simulation and a partial efficiency gain, provided there is little variation in the externally forced temperature component of $x$ or $z$ and in the weight $w$ within units. Arguments for short units are the anticipated withinunit variation in the forced component and (sometimes) in $w$, together with the need to estimate sample variances (see Sect. 5 in SUN12). The latter can be problematic, in particular, because the length of the available instrumental record poses an upper limit on the length of time units that can be used. For example, with 120 years of instrumental observations, only four samples would be present for estimation of the instrumental temperature variance if the chosen time unit were 30 years. We have aimed to make time units short while controlling the autocorrelation. 

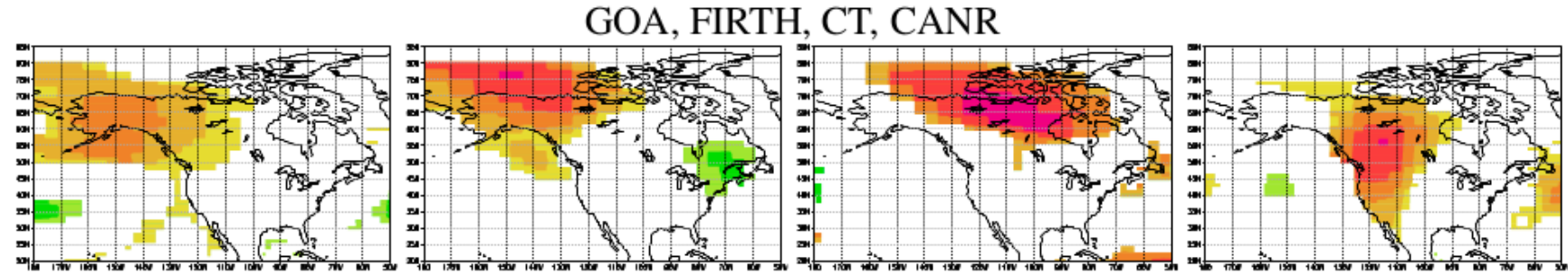

TORN, JAMT, TATRA, ALPS, PYR
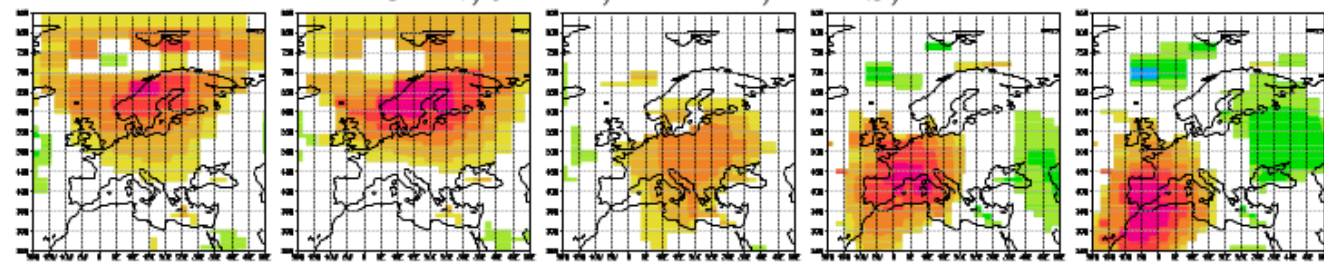

YAMC, AVAMT, YAK, ASIA2K
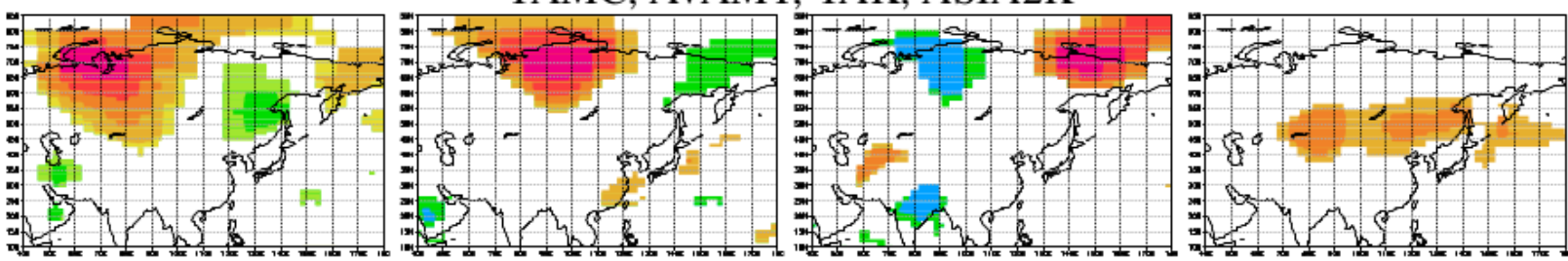

TASM, NZ
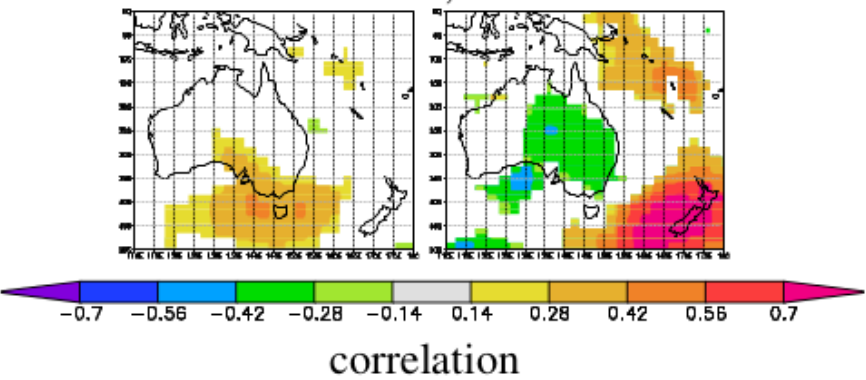

Figure 1. Correlation between each tree-ring chronology and the GISS1200 instrumental temperature field, based on first-differenced data for seasonal averages and time periods as used for calibration by each original investigator (see Tables 1 and 3). Colours are muted where correlations are not significant at the $5 \%$ level. Analysis made on the KNMI Climate Explorer (http://climexp.knmi.nl, Trouet and van Oldenborgh, 2013).

The shortest possible time unit is dictated by the resolution of tree-ring data, which is 1 year. Thus, letting the time unit be 1 year would maximize the sample size. Therefore, we have always used the 1-year unit for calibration of the tree-ring records (in Sect. 4.5). However, before calculating $U_{R}$ and $U_{T}$ statistics, we need to check that the choice of time unit there will not seriously violate the assumption that unforced temperature variability can be approximated by an AR(1) process (see Appendix A).

To determine this, we analyse the autocorrelation in the 3000-year control simulation in two ways for each region. First, the lag-1 autocorrelation is computed for all time units from 1 to 30 . Then, for a few selected time units $(1,3,5,8$, 12 years), the autocorrelation function is estimated for lags up to 30 . Figure 4 suggests that the lag- 1 autocorrelation is in agreement with white noise except in some regions at short time units. Further, Fig. 5 (top) reveals that an $\operatorname{AR}(1)$ process is not sufficient at the 1-year unit within four regions (GOA, ASIA2k, TASM, NZ), which show a clear oscillatory behaviour with a period of about 3-4 years. As these four regions are located near the Pacific Ocean, a reasonable guess is that the model's El Niño-Southern Oscillation could be the cause. For the other four selected time units (Fig. 5, middle and bottom), we find support for either a white noise or an $\mathrm{AR}(1)$ assumption. Thus, for this study, we choose time units of 3, 5, 8 and 12 years to compute $U_{R}$ and $U_{T}$ statistics and compare the results. We use the $A R(1)$ adjustment from Appendix A whenever the estimated lag-1 autocorrelation is positive. Although negative lag-1 autocorrelations may be physically meaningful in some cases (see e.g. Vyushin et al., 


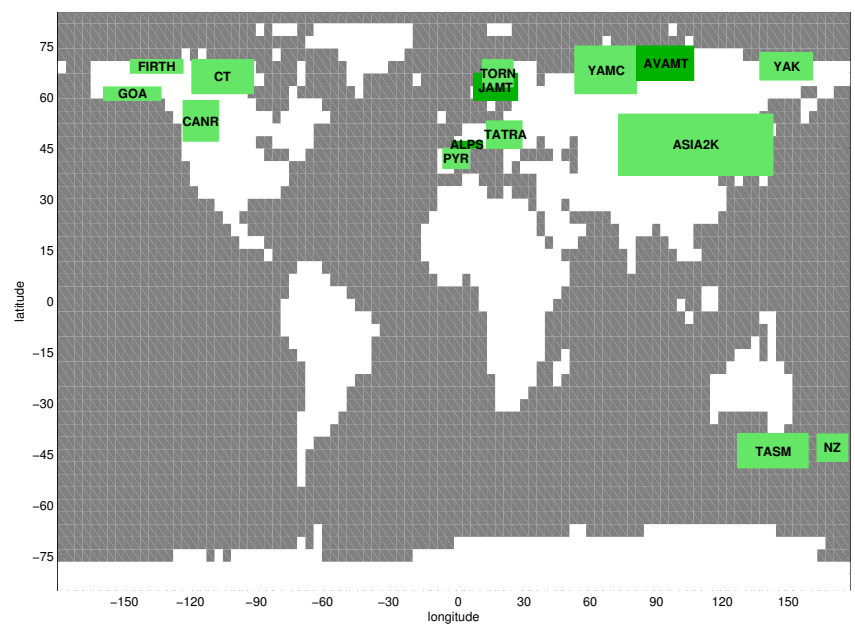

Figure 2. Location of regions that the 15 tree-ring records represent, plotted on the land/sea mask of the MPI-ESM model. Regions' short names are explained in Table 1.

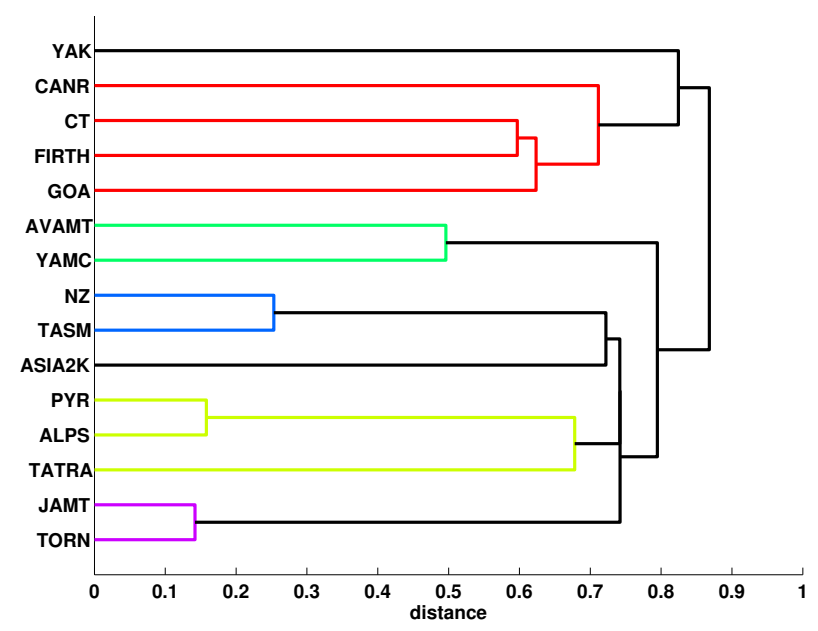

Figure 3. Hierarchical cluster tree based on nearest-neighbour linkage with $(1-r)$ as distance metric, where $r$ is the sample correlation. Data from the 3000-year-long unforced control simulation, for seasons as specified in Table 1, are used for the cluster analysis.

2012), we assume white noise is reasonable whenever estimated values are negative. Hence, we are on the safe side since negative lag- 1 correlation is associated with a reduced standard deviation of the $T$ statistics. Moreover, when comparing two forced simulations directly, as in Appendix B, we also need to check that the forced simulated temperatures do not violate the AR(1) assumption. Thus, as we use this new approach to compare the low and high solar ensembles (E1 and E2 simulations), we also made the same checks for those data (results are not shown). As expected, we found evidence for stronger lag-1 autocorrelation than in the unforced control simulation, but an AR(1) assumption is valid in the large majority of cases. A few regions in the E2 (high solar) simulations showed more persistence than expected from $\operatorname{AR}(1)$, but this does not affect our results since only E1 (low solar) data are used to estimate the autocorrelation (see Appendix B4).

\section{Results and conclusions from calculation of $U_{R}$ and $U_{T}$ statistics}

Figure 6 shows calculated $U_{R}$ and $U_{T}$ statistics for individual regions and when all regions are combined in different ways, for the four selected time units. Notably, none of the single-forcing simulations robustly show $U_{R}$ values above the $5 \%$ significance threshold. Only the volcanic simulation shows some (barely) significant $U_{R}$ values for the combined regions, but only for one or two time units. Regionally combined $U_{R}$ values for each individual simulation in the E1 (low solar) multiple-forcing ensemble are often, but not always, above the $5 \%$ significance threshold. The corresponding values for the E2 (high solar) ensemble are higher and always above the significance threshold. Regionally combined $U_{R}$ values for the ensemble averages are significant for both the low and high solar ensembles (E1 and E2). This holds for all four regional weightings - with $p$ values actually much smaller than 0.05 .

These results provide some general information. First, they illustrate how the combination of several forcings contributes to give significant correlations with the observed temperature variation, despite the often non-significant results for the individual forcings alone. Note, however, that also greenhouse-gas and orbital forcings are included in the E1 and E2 simulations, although no single-forcing simulations are available with these two forcings. Therefore, we cannot judge how much the latter contribute to the significant test values. Second, the variation among $U_{R}$ values between individual members in the E1 and E2 ensembles illustrates the degree of randomness that is due solely to different initial conditions among the simulation ensemble members. This is essential to bear in mind when considering results where no such ensemble is available, such as for the solar and volcanic single-forcing simulations used here. Third, the results show that the forced component in simulated temperatures stands out more clearly in an ensemble average than in a single simulation. This holds for both E1 and E2, but the effect should be strongest for the larger E1 ensemble size.

We can conclude that both multiple-forced ensembles explain a statistically highly significant proportion of the temporal variation seen in tree-ring data. Thus, it is a meaningful exercise to see whether they also fit the observations better than unforced simulations. Figure 7 is an attempt to graphically illustrate how well the low and high solar ensemble (E1 and E2) simulation time series match the tree-ring-based observations and how they compare with the unforced control simulation. Although $U_{R}$ and $U_{T}$ values are calculated separately for each region, the figure for simplicity shows data averaged over all regions (and only for the 12-year unit). By 


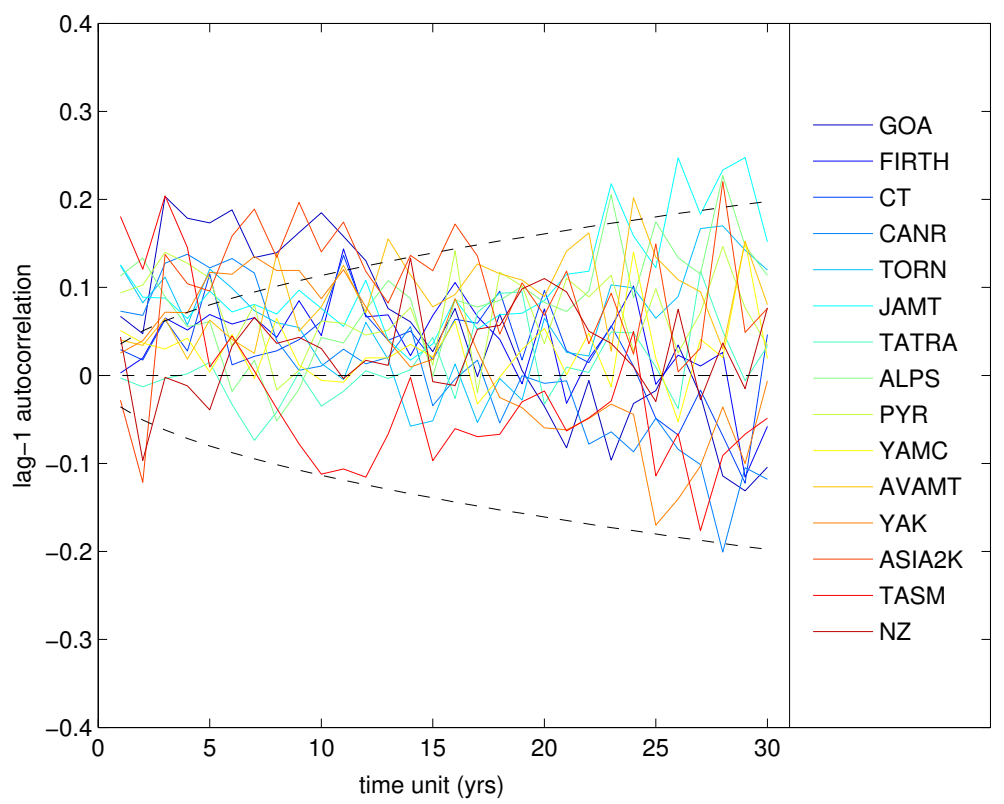

Figure 4. Estimated lag-1 autocorrelation for time units from 1 to 30 years in the 3000-year-long unforced control simulation. Two-sided $5 \%$ significance levels for a white noise process are shown with dashed lines. Data for each region, identified by the colour legend to the right, are for the season as specified in Table 1.

eye, one can see a correlation between proxy data and both types of multiple-forced simulations, but it is not easy to see whether these forced simulations are significantly closer to the observed temperature variations than the control simulations. This, however, is precisely what the $U_{T}$ statistic can tell. It turns out that the ensemble $U_{T}$ values for the combined regions are negative for both the low (E1) and high (E2) solar ensembles (i.e. they are plotted above the zero line in Fig. 6), although this does not hold for some individual simulations within the ensembles. Thus, when the entire multiple-forced ensembles are considered, both of them show smaller calculated $D_{\mathrm{w}}^{2}$ distances to the tree-ring-based observations than if unforced simulations are used. However, ensemble $U_{T}$ values are not always significant at the 5\% level - but they are significant for some time units, or regional weightings, for both high and low solar ensembles. Because the two ensembles have different size, their $U_{T}$ statistics calculated in this way should not be compared to judge whether one of them fits the observations better than the other. But this can instead be tested by using the relaxed assumption in Appendix B, which permits the computation of $U_{T}$ to test directly whether one of the two simulation ensembles is significantly closer to the proxy data than the other.

Figure 8 shows these $U_{T}$ values for all four time units. Negative values (upwards) indicate where the high solar (E2) ensemble is closer to the observed temperatures, whereas positive values (downwards) indicate where the low solar (E1) ensemble is closer. The $U_{T}$ values obtained when regional information is combined are always insignificant. In most cases, the individual regional $U_{T}$ values are also not statistically significant, but some significant values of both negative and positive sign are found.

Clearly, neither of the E1 (low) and E2 (high) solar ensembles is significantly closer to the observed temperature variations than the other. Moreover, results vary between regions. As concerns the effect of including or excluding the three tree-ring records where RCS was not used, the regionally weighted results change very little. However, it may be noted that the non-RCS GOA record provides significant positive $U_{T}$ values in three of the four cases illustrated in Fig. 8, whereas the relatively nearby non-RCS CT record always provides negative $U_{T}$ values, of which one is significant. This further underscores the large variation of results among the regional data series.

\section{Final discussion and conclusions}

Practical application of the SUN12 framework (Sundberg et al., 2012) and certainly also other methods for palaeoclimate model-data comparison, e.g. in data assimilation or detection and attribution studies, involve several decisions to be made by the investigator. A possible solution to handle this situation is to make a few alternative decisions and study how sensitive the results are. This approach is relevant in the current study, which is mainly methodological in nature. We studied the effect particularly related to two decisions: the choice of weighting information from different regions and the choice of time unit (time resolution). The latter was facilitated by an improvement in the framework to allow un- 

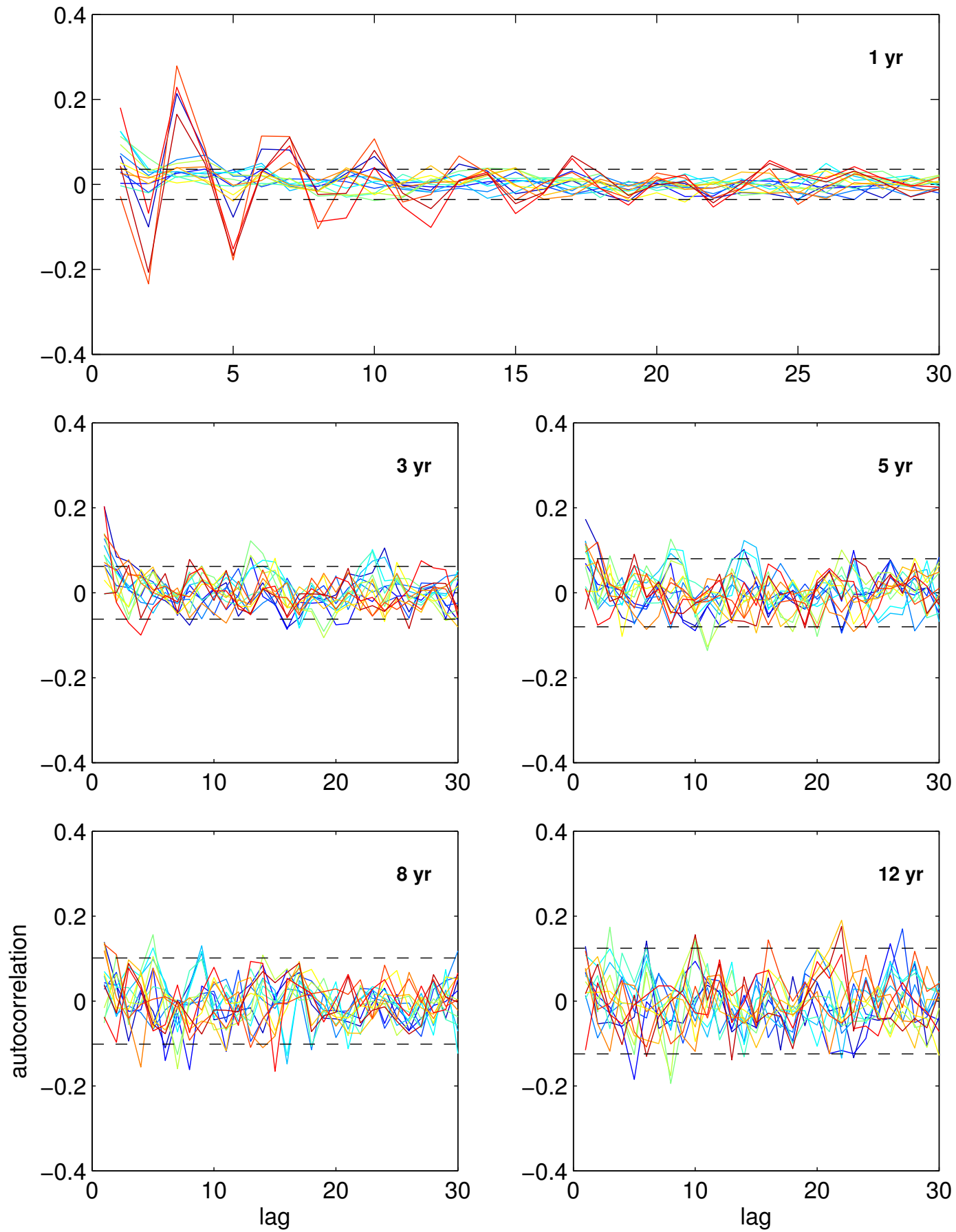

Figure 5. Estimated autocorrelation function for lags up to 30 in the 3000-year-long unforced control simulation, for time units of 1, 3, 5, 8 and 12 years. Two-sided $5 \%$ significance levels for a white noise process are shown with dashed lines. Data for each region, identified by the colour legend to the right in Fig. 4, are for the season as specified in Table 1.

forced simulated temperatures to follow an AR(1) process rather than just white noise as in SUN12. This made it possible to choose time units down to 3 years, which is considerably shorter than the 20 or 30 years used in earlier studies by Hind and Moberg (2013) and Hind et al. (2012). Although an AR(1) assumption was empirically found valid for climate model data at time units used here, it could be motivated with further development of the framework to also account for the possibility that simulated climate shows stronger persistence, such as a power law, as has been found by, for example, Vyushin et al. (2012). 

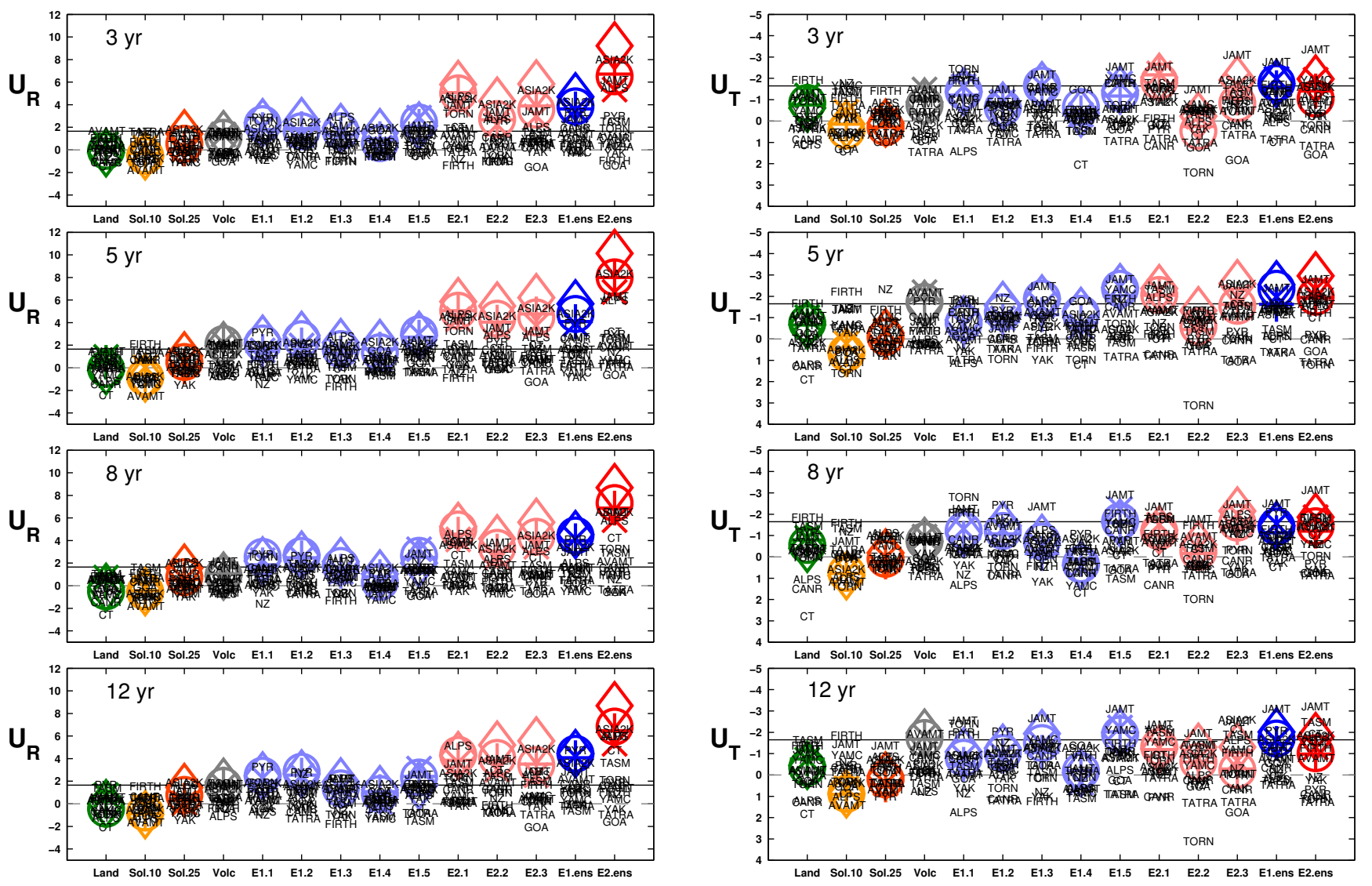

Figure 6. $U_{R}$ and $U_{T}$ statistics from comparisons between simulated temperatures and tree-ring-based temperature observations in the period 1000-1849 CE, for time units of 3, 5, 8 and 12 years. Results are shown for single-forcing simulations (land use, low-amplitude solar, highamplitude solar, volcanic), each individual simulation in the E1 and E2 multiple-forcing ensembles (including low and high solar forcing, respectively), and for the whole $\mathrm{E} 1$ and $\mathrm{E} 2$ ensembles. $U_{R}$ and $U_{T}$ values for each region are denoted with site short names. Results where all sites are combined are shown with symbols to distinguish between different $c_{j}$ weightings $(\bigcirc$ equal, $\diamond$ area, + cluster, $\times$ equal without non-RCS series). Solid lines show $5 \%$ significance levels. Note the reversed vertical axis in the $U_{T}$ graphs.

In this study, we used an ensemble of climate model simulations run with forcing conditions for the last millennium (Jungclaus et al., 2010), which we compared with a set of 15 tree-ring-based temperature proxy data series representing regions of different size, different seasonal mean temperatures and with different lengths and statistical precision. Our results showed that, among the single-forcing simulations (land use, small-amplitude solar, large-amplitude solar, volcanic), only the one with volcanic forcing could, with statistical significance, explain any of the observed variations in the pre-industrial period 1000-1849 CE - but only for one or two of four time units tried, depending on which regional weighting was used. Preferably, ensembles of simulations with single forcings would be needed to study whether this result is robust to randomness associated with simulated internal (unforced) variability. Nevertheless, we note that this finding - that only the effect of volcanic forcing, but not solar forcing, could be significantly detectable in proxy data is in agreement with results from detection and attribution studies both at a hemispheric scale (Hegerl et al., 2007) and a European scale (Hegerl et al., 2011). A more recent detection and attribution study (Schurer et al., 2014) confirms that volcanic (and also greenhouse gas) forcing seems to have an important influence on temperature variability in the period 1000-1900 CE, while the contribution from solar forcing was found to be modest.

When all forcings were combined (land use, smallamplitude or large-amplitude solar, volcanic, orbital, greenhouse-gas) and also used in small simulation ensembles, the simulations were, however, highly able to capture some of the observed temperatures as recorded in tree-ring data. The reasons behind the significant test values are partly due to the combination of the effect from several forcings and partly because the response to forcings is expected to stand out more clearly in an ensemble average than in a single simulation, as has been demonstrated previously in pseudoproxy experiments (Hind et al., 2012) and here using tree-ring temperature proxy data. Both multiple-forced simulation ensem- 

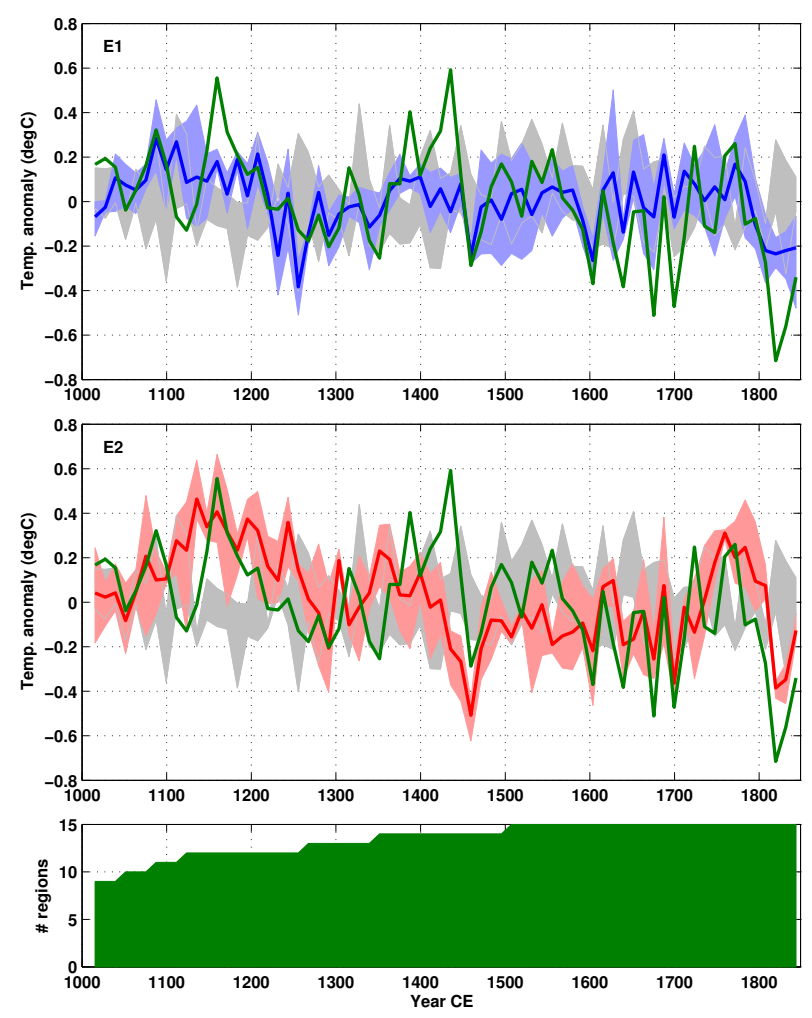

Figure 7. Time series illustration of data used for the $U_{R}$ and $U_{T}$ analysis at the 12-year time unit. Green curves show the arithmetic average of all calibrated proxy series ( $z$ series). Blue and red curves show the corresponding values for simulated multiple-forced temperatures ( $x$ series), averaged over the low solar (E1, blue) and high solar (E2, red) ensemble members. Light-blue and light-red bands show the range between the highest and lowest regionally averaged simulated temperatures within the E1 and E2 ensembles. Grey bands show the corresponding range for the control simulation ensemble. Temperatures are shown as anomalies with respect to long-term averages, as used for the $U_{R}$ and $U_{T}$ calculations. The bottom graph shows how the number of regions change with time.

bles are closer to the tree-ring-based observations than if unforced control simulations are used, implying that the temperature response to the combination of forcings is realistic. However, results at the individual regional level differ greatly and significance is not reached for all time units and choices of weights when regions are weighted together. A conclusion here is that an average of many sites is needed, or the separate sites/records need to be more clearly classified as less or more reliable and representative than others.

Another improvement to the SUN12 framework made it possible to test directly whether one of the two multipleforced simulation ensembles (i.e. including either small- or large-amplitude solar forcing) is closer to the observed temperature variations than the other. However, results were highly variable at the regional level, which made it impossible to judge whether any simulation ensemble is more realistic than the other. Thus, this new analysis based only on tree- ring data from several regions did not show any clearer results than a previous northern hemispheric-scale study based on several compilations of different proxy data (Hind and Moberg, 2013) ${ }^{3}$. This inconclusiveness is perhaps not surprising given that differences between simulations with frequently used weaker or stronger solar forcing are rather small (Masson-Delmotte et al., 2013).

Although the weaker solar forcing is more in line with most recent viewpoints (Masson-Delmotte et al., 2013; Schurer et al., 2014), it is still possible that none of the two alternative solar forcings used here is correct and that the truth is somewhere in between. An extension of the framework to allow estimation of how well the amplitude of a true external forcing is represented in a simulation could perhaps help to provide a more informative answer. As already argued in SUN12 (Sect. 9), such an extension would also bring their framework closer to that used in detection and attribution studies (e.g. Hegerl et al., 2007; Schurer et al., 2013, 2014).

One may ask as to what extent the choice of using only tree-ring data has influenced the results. For example, their inability to correctly capture the long-term trend on millennial scales has been discussed by Esper et al. (2012). Such a long-term temperature trend is expected to result from the slowly changing orbital climate forcing, which is large in extratropical regions within the growing season (see e.g. Phipps et al., 2013). This problem should be most prominent in records where RCS standardization was not used. Omitting the three non-RCS records in our collection, however, did not change the main result. Instead we noted that the individual non-RCS records could give opposite results regarding the question of whether the low or high solar ensemble is closer to the observations. This further accentuates that many proxy sites are needed in order to obtain robust results. We have also argued that variance stabilization procedures (Osborn et al., 1997) applied to many tree-ring chronologies are in conflict with assumptions in the SUN12 framework. This may affect results, presumably making statistical test values too high. Another potential problem is the observed spectral biases in many tree-ring records (they are often too "red"; Franke et al., 2013). This does not affect the validity of the tests, but will affect their power. It remains to analyse how these and other problems, e.g. regarding the different nature of response in TRW and MXD data to volcanic forcing (D'Arrigo et al., 2013; Esper et al., 2013; Jones et al., 2013), affect results from model vs. tree-ring data comparisons.

Deficiencies in the climate model may also influence the results. The model used in this study is a low top model (Charlton-Perez et al., 2013) without interactive ozone and with the solar variation implemented only by modulating

\footnotetext{
${ }^{3}$ Hind and Moberg (2013) used the inside averaging method, which we do not use in the present study. Therefore, we have now repeated their experiment but using instead the outside averaging method. None of their main conclusions are affected by this change.
} 


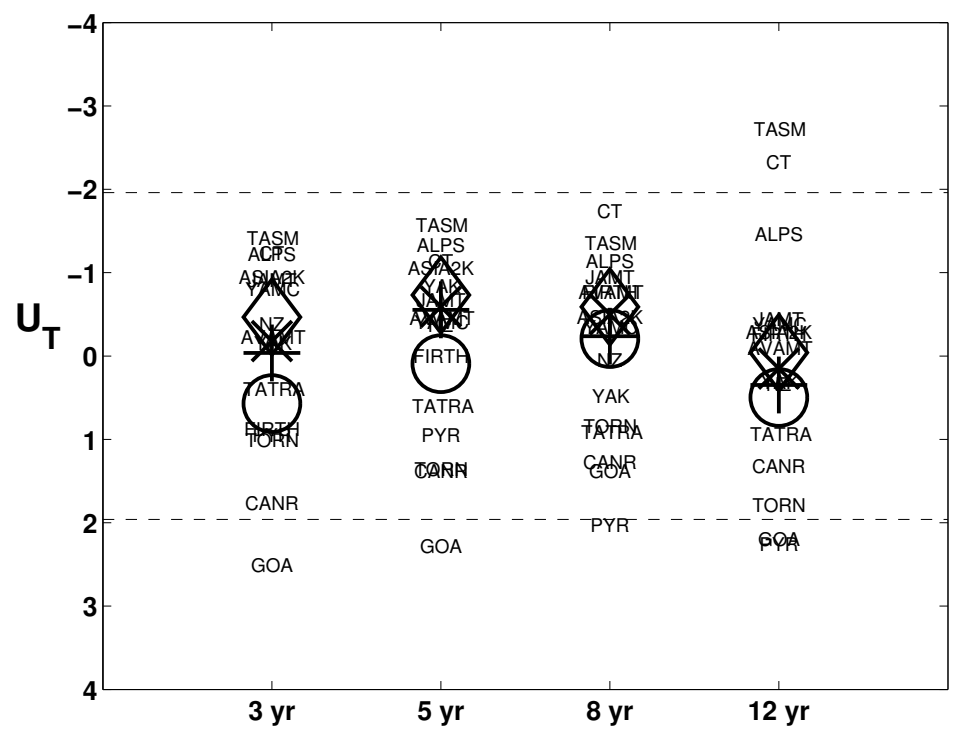

Figure 8. $U_{T}$ statistics comparing the multiple-forcing simulation ensembles with low and high solar forcing amplitude. Negative values (upwards) indicate where the high solar (E2) ensemble is closer than the low solar (E1) ensemble to the tree-ring-based observations. Symbols show results where all regions are combined with different $c_{j}$ weightings $(\bigcirc$ equal, $\diamond$ area, + cluster, $\times$ equal without non-RCS series). Results are shown for four time units. Dashed lines show $5 \%$ significance levels for testing the null hypothesis that no simulation ensemble is closer to the observations than the other.

the total solar irradiance (not the spectral irradiance). It is therefore possible that it may lack possible dynamical responses (see e.g. Gray et al., 2010) and the highly variable regional results may reflect such deficiencies of the climate model. Moreover, these model simulations have an interactive carbon cycle, which, although the model hopefully becomes more realistic than if a prescribed $\mathrm{CO}_{2}$ forcing is used, can induce an increased potential source of error. Here, this leads to somewhat different time evolution of the simulated $\mathrm{CO}_{2}$ concentrations in the two ensembles, where both show discrepancies compared to the reconstructed $\mathrm{CO}_{2}$ concentrations (see Fig. 6 in Jungclaus et al., 2010). This might affect our results in one direction or the other. An additional reason for the inconclusive results could be the small ensemble size for the simulations; the signal-to-noise ratio would increase when averaging over a larger ensemble.

Information also from other types of proxy data should potentially help to more conclusively compare a set of alternative simulations with proxy-based climate observations. All our proxy records reflect temperatures only in the treegrowth season, i.e. mostly a summer or an extended summer season. Perhaps the regions used here are too small, or too few, or not sufficiently well distributed in space. In combination with a lack of information from winter, this might cause internal unforced variability to dominate too much over the response to external forcings. A model study by Servonnat et al. (2010) suggested that the responses to external forcings are only detectable within regions larger than approximately the size of Europe, thus pointing to the importance of not using regions that are too small in studies like this. On the other hand, the pseudoproxy study by Hind et al. (2012) suggested that annual-mean temperature data, with realistic proxy noise levels, from at least 40 randomly distributed single grid-boxes are needed to clearly separate between the two sets of multiple forcings used here. Thus, averaging information from a sufficient number of small regions can be meaningful, even if each region by itself is too small to clearly separate the externally forced signal from internal climate variability.

There are certainly many more published proxy records (and more are expected to appear in the future) that could potentially be used in this type of model-data comparison study. But it is still somewhat open regarding whether proxy data are best used as individual records, as is the case with most records in this study, or aggregated into larger-scale averages such as in the PAGES2k data set (PAGES2k Network, 2013). In that study, seven continental-scale annualmean or summer-mean temperature reconstructions (including ASIA2 $k$ used here) were derived from different types of proxy data. This latter approach has the potential advantage of reducing the influence from various types of noises, both in proxy data and from internal variability in both models and real climate. A drawback, though, is that seasonally specific information in each proxy is partially lost and the optimal region and season for each large-scale data aggregate is essentially unknown. Thus, more theoretical and practical work addressing questions such as the optimal spatial analysis scale is motivated - in parallel with continued development of climate models, forcing data sets and climate proxy records. 


\section{Appendix A: Adjustment for autocorrelation in the reference climate model simulation series}

This is a derivation of an adjustment factor for the correlation test statistic $R$ and for the $D^{2}$-based test statistic, necessary to allow autocorrelation in the reference climate model simulation series, in particular under an AR(1) model for this autocorrelation. Finally, an MA(1) model and the effects of a $k$-years time unit are also treated.

\section{A1 Model}

Suppose we have a climate model ${ }^{4} x$ with time-varying forcing and another, $x^{*}$, as a reference free from such forcings. We also have an observation series for the same period as the forced model, denoted $z$. The observations $z_{i}$ (time step $i=1, \ldots, n$ ) represent instrumental measurements when such are available, or otherwise a proxy assumed to be correctly calibrated. We want to test first whether the forced model $x$ shows evidence of a correlation with the observations ( $R$ test), and next whether it fits the observations better than the reference model $x^{*}\left(D^{2}\right.$-based test). Of concern here is the performance of the test statistics when there is autocorrelation present in both climate models. To represent the hypothesis model $H_{0}, x$ and $x^{*}$ are taken to be mutually equivalent (because $H_{0}$ implies lack of forcing effect) and autocorrelated, but uncorrelated with the true and measured temperatures, $\tau$ and $z$ :

Statistical model under $H_{0}$ : Climate model simulation sequences $\left\{x_{i}\right\}$ and $\left\{x_{i}^{*}\right\}$, true climate sequence $\left\{\tau_{i}\right\}$, and observation sequence $\left\{z_{i}\right\}$ are described by the following stationary model:

$x_{i}=\mu_{x}+\delta_{i}, \quad \operatorname{Corr}\left(\delta_{i}, \delta_{i-k}\right)=\rho_{k}$,

$x_{i}^{*}=\mu_{x}+\delta_{i}^{*}, \quad \operatorname{Corr}\left(\delta_{i}^{*}, \delta_{i-k}^{*}\right)=\rho_{k}$,

$\tau_{i}=\mu_{\tau}+\eta_{i}$,

$z_{i}=\tau_{i}+\epsilon_{i}$.

Note that the model treats $x$ and $x^{*}$ the same, the reason being that there is no forcing effect in this $H_{0}$ model. The test statistics, however, were designed to be sensitive to a forcing effect that $x$ and $\tau$ have in common. The variates $x_{i}$ and $x_{i}^{*}$ have the same mean value $\mu_{x}$, and mutually independent "noise terms" $\delta_{i}$ and $\delta_{i}^{*}$. Other terms representing unexplained variability (random fluctuations, internal variability, noise) are $\eta_{i}$ and $\epsilon_{i}$. Here $\eta_{i}$ represents the true climate variability around the mean value $\mu_{\tau}$, including the possible forcing effect on the true climate (which we hope to find in $x$

\footnotetext{
${ }^{4}$ With "climate model", we think of a realization of an atmosphere-ocean general circulation model or an Earth system model integrated in time, with or without time-varying external forcings. The variable $x$ represents simulated temperatures in a certain region and season of interest.
}

but assume under $H_{0}$ to be missing in $x$ ). We need not make any specific assumption about that variability. In particular, autocorrelation is allowed in the $\tau$ and $z$ sequences without need for adjustment. Technically, the observed $z$ series is regarded as given and fixed, and the statistical properties of the test statistics are conditional on this given series. Weights $w_{i} \geq 0$ and $\widetilde{w}_{i} \geq 0$ (see Sects. 5 and 8 in SUN12) are also regarded as given and fixed. Concerning the definition of $\widetilde{w}_{i}$, note the footnote in Sect. 2 of the current study.

We consider the correlation test and the $D^{2}$-based test, based on the same basic statistics as in the absence of autocorrelation, but we have to modify their variances (or standard errors) in order to allow autocorrelation. For the correlation test we do not need the unforced climate model, since the hypothesized correlation is known, being zero.

\section{A2 Correlation test statistic}

The weighted empirical regression coefficient $R(x, z)$ is used as a test statistic, after normalization by its standard error (see Eqs. 19 and 20 in SUN12). Now, $R(x, z)$ differs only by a constant factor from the weighted covariance $\sum \widetilde{w}_{i}\left(x_{i}-\right.$ $\left.\mu_{x}\right) z_{i}$. We need an expression for its variance, allowing some degree of autocorrelation.

First we note that, since $\widetilde{w}_{i}$ and $z_{i}$ are both regarded as fixed and given quantities, we may introduce a new weight factor $\dot{w}_{i}=\widetilde{w}_{i} z_{i}$, which is their product. Thus, we consider the variance of

$\sum \dot{w}_{i}\left(x_{i}-\mu_{x}\right)$

or, equivalently,

$\sum \dot{w}_{i} x_{i}$

We start by the general formula for the variance of a linear expression,

$$
\begin{array}{r}
\operatorname{Var}\left(\sum_{i} \dot{w}_{i} x_{i}\right)=\sigma_{x}^{2} \sum_{i, j} \dot{w}_{i} \dot{w}_{j} \rho_{|j-i|} \\
=\sigma_{x}^{2}\left(\sum_{i} \dot{w}_{i}^{2}+2 \sum_{i<j} \dot{w}_{i} \dot{w}_{j} \rho_{j-i}\right) .
\end{array}
$$

If we know the autocorrelations, this exact value can be used. Here we continue by assuming that the $x_{i}$ time series is a stationary $\mathrm{AR}(1)$ process with lag-1 correlation $\rho$ :

$x_{i}-\mu_{x}=\rho\left(x_{i-1}-\mu_{x}\right)+\widetilde{\delta}_{i}, \quad|\rho|<1$,

where $\widetilde{\delta}$ is a white noise error term. In such a model, the lag $j-i$ correlation $\rho_{j-i}$ for $j \geq i$ decreases exponentially with the time distance $j-i, \rho_{j-i}=\rho^{j-i}$. We may then rewrite Eq. (A1) as

$$
\operatorname{Var}\left(\sum_{i} \dot{w}_{i} x_{i}\right)=\sigma_{x}^{2}\left(\sum_{i} \dot{w}_{i}^{2}+2 \sum_{k=1}^{n-1} \rho^{k} \sum_{i ; i>k} \dot{w}_{i} \dot{w}_{i-k}\right) .
$$


This exact value can be used, but we will also give a simple upper bound to it that we have used in this paper. We first assume $\rho \geq 0$, which appears realistic if $x_{i}$ is $\operatorname{AR}(1)$. We use Cauchy's inequality $\sum_{i>k} \dot{w}_{i} \dot{w}_{i-k} \leq \sum_{i} \dot{w}_{i}^{2}$ to get the upper bound

$$
\begin{aligned}
\operatorname{Var}\left(\sum_{i} \dot{w}_{i} x_{i}\right) & \leq \sigma_{x}^{2} \sum_{i} \dot{w}_{i}^{2}\left(1+2 \sum_{k>1} \rho^{k}\right) \\
& \leq \sigma_{x}^{2} \sum_{i} \dot{w}_{i}^{2} \frac{1+\rho}{1-\rho} .
\end{aligned}
$$

The last inequality is obtained when the finite sum of $\rho^{k}$ up to $k=n-1$ is majorized by the corresponding infinite sum.

Thus we have an upper bound for the variance as a function of $\rho$. The variance factor

$$
(1+\rho) /(1-\rho)
$$

is what differs from the case $\rho=0$. This formula was derived under the assumption $\rho \geq 0$. When $\rho<0$, factor (A4) can be replaced by the value 1 , so no adjustment is needed. For the standard error, we use of course the square root of factor (A4). For $\rho$ small, the variance factor (A4) is about $1+2 \rho$, or for the standard error $1+\rho$, but this approximation is no longer an upper bound, so $(1+\rho) /(1-\rho)$ is preferable.

The second inequality above, where the finite sum was replaced by an infinite sum, should typically be very close to an equality. On the other hand, the inequality motivated by Cauchy's formula is likely to be a large exaggeration of the actual value. There are two parts in this inequality. First, the sum of squares, $\sum_{i} \dot{w}_{i}^{2}$, contains $n$ terms, whereas the sum of products, $\sum_{i>j} \dot{w}_{i} \dot{w}_{i-j}$, contains only $n-j$ terms. However, since the contributions from $\rho^{j}$ with small $j$ are likely to dominate, this will make little difference.

The second part concerns the magnitude of sums of products relative to the sum of squares. Here we must bring in the structure of the $z$ series of real climate plus noise. A sum of products relates to the covariance of the $z$ series, and if there is no very high autocorrelation in the $z$ series, the sum of products will be much smaller than the sum of squares (representing the variance). Thus, multiplying the variance in Eq. (20) in SUN12 by the factor $(1+\rho) /(1-\rho)$ is likely to substantially exaggerate the effects of an $\mathrm{AR}(1)$ autocorrelation in the $x$ series. Nevertheless, this is how we made the adjustments in this study. When data from different regions are combined, an adjustment factor has to be calculated for each region separately. Then, the covariances in Eq. (21) in SUN12 are multiplied by the square root of the product of the pairs of adjustment factors.

\section{A3 $D^{2}$ difference test statistic}

Here we investigate the effects of autocorrelation on the $D^{2}$ difference test statistic $T\left(x, x^{*}, z\right)=D_{\mathrm{w}}^{2}(x, z)-D_{\mathrm{w}}^{2}\left(x^{*}, z\right)$ (see Eq. 1). By expanding the quadratics of $x_{i}-z_{i}$ and of $x_{i}^{*}-z$, it is seen that $T$ can be expressed in the form

$$
\begin{aligned}
T\left(x, x^{*}, z\right) & =\overline{w\left(x-\mu_{x}\right)^{2}}-\overline{w\left(x^{*}-\mu_{x}\right)^{2}} \\
& -2 \overline{w\left(x-x^{*}\right)\left(z-\mu_{x}\right)},
\end{aligned}
$$

with overlines denoting averaging over time ( $n$ time steps). Because $x$ and its corresponding reference $x^{*}$ are equivalent under $H_{0}$, the first two terms have equal expected values and the third term will have an expected value of zero, so $T$ has an expected value of zero. Autocorrelation in $x$ and $x^{*}$ does not change this. To specify a test statistic we only also need the variance of $T$ in the hypothesis model. This will be influenced by autocorrelation.

The first two terms of Eq. (A5), right-hand side, are mutually uncorrelated, since $x$ and $x^{*}$ are mutually independent. Each of them is also uncorrelated with the third term, under an assumption of Gaussian noise $\delta$ and $\delta^{*}$ in $x$ and $x^{*}$, respectively (already made in SUN12). This is because the covariances will be proportional to the third-order central moments of $\delta$ (or $\delta^{*}$ ), which are zero because of the symmetry of the Gaussian distribution around its mean value (the only property of the Gaussian needed, in fact). Note that $x-x^{*}=\delta-\delta^{*}$. Thus, all three terms are mutually uncorrelated, so we need only consider the sum of their respective variances.

The last term is linear in $x$, and it is the difference between two mutually uncorrelated expressions of the same type as the statistic studied in Appendix A2. By referring to the same argument as there, we conclude that a safe variance adjustment factor is $(1+\rho) /(1-\rho)$. It is again likely to be a relatively crude upper bound. Like in Appendix A2, it is possible to use an exact expression for that term as an alternative.

Turning to the first two terms, note that they are of identical type, so we need only study one general such statistic,

$\sum w_{i}\left(x_{i}-\mu_{x}\right)^{2}$.

Note that $z$ is not involved here, so the weight factor is the original relatively slowly varying $w_{i}$, not the $\dot{w}_{i}$ of Appendix A2. Under the AR(1) model (A2), we have

$$
\left(x_{i}-\mu_{x}\right)^{2}=\rho^{2}\left(x_{i-1}-\mu_{x}\right)^{2}+2 \rho\left(x_{i-1}-\mu_{x}\right) \widetilde{\delta}_{i}+\widetilde{\delta}_{i}^{2} .
$$

It follows that the covariance between $\left(x_{i}-\mu_{x}\right)^{2}$ and the corresponding preceding term is

$$
\operatorname{Cov}\left\{\left(x_{i}-\mu_{x}\right)^{2},\left(x_{i-1}-\mu_{x}\right)^{2}\right\}=\rho^{2} \operatorname{Var}\left(\left(x_{i-1}-\mu_{x}\right)^{2}\right) .
$$

This is because $\widetilde{\delta}_{i}$ and $x_{i-1}$ are mutually independent. Repeating this step back in time we get

$$
\operatorname{Cov}\left\{\left(x_{i}-\mu_{x}\right)^{2},\left(x_{i-k}-\mu_{x}\right)^{2}\right\}=\rho^{2 k} \operatorname{Var}\left(\left(x_{i-k}-\mu_{x}\right)^{2}\right) \text {. }
$$

Now we can do the same type of calculation as for the linear type of term in Appendix A2, but with $\rho^{2}$ replacing $\rho$, and 
get the variance adjustment factor

$\frac{1+\rho^{2}}{1-\rho^{2}}$.

Note that the replacement of $\rho$ by $\rho^{2}$ makes this adjustment factor much closer to 1 . On the other hand, this upper bound is not likely to exaggerate much the actual variance increase. This is because in contrast to $\dot{w}_{i}, w_{i}$ will mostly change slowly with $i$, implying $\sum w_{i} w_{i-k} \approx \sum w_{i}^{2}$ for small $k$.

There are two possible strategies when choosing the adjustment factor. Either we simply use formula (A4) for the whole variance of $T$, which is then a deliberate overadjustment, or we split $T$ into its three components and use their respective variances with different adjustments for the different components. In our applications we have used the first (simpler) alternative. Thus, the variance in Eq. (15) in SUN12 has been multiplied by the factor (A4). When data from different regions were combined, we calculated one adjustment for each region. Then, the covariances in Eq. (16) in SUN12 were multiplied by the square root of the product of the pairs of adjustment factors. In other words, we used the same adjustment for the correlation and the difference tests.

\section{A4 Autocorrelation and time units}

The results above were derived under an AR(1) model for the unforced climate simulations. Figure 5, top, shows the corresponding estimated autocorrelation functions for our annual data. Even if some regions appear consistent with the exponentially decreasing autocorrelation function of an AR(1), other regions show a damped sine-wave-type function, indicating an $\mathrm{AR}(2)$ process, or worse. The damping factor is of magnitude 0.85 year $^{-1}$. An AR(2) model would make the previous calculations considerably more complicated. Going further away from AR(1) by using a model with long-range dependence will change the situation completely.

Hind et al. (2012) used a longer time unit to make all correlation negligible. With a too short time unit, the lag-1 correlation is not negligible. However, because of the time gap between time units at lag $\geq 2$ distance, correlations for lag-2 (or more) are likely to be much smaller than as prescribed by $\operatorname{AR}(1)$ (which is $\rho^{2}$ ). As a numerical example, suppose we have an $\operatorname{AR}(1)$ for annual data with $\rho=0.45$. If we change time unit to 3 years, lag- 1 correlation is of course reduced, but of interest here is that the lag- 2 correlation is reduced much more, becoming a factor $\rho^{3} \approx 0.1$ times lower than the corresponding lag-1 correlation. More generally, for a series whose autocorrelations in the moderately long run decrease like in an $\operatorname{AR}(1)$ series, only a moderately long time unit is needed to make all lag $\geq 2$ autocorrelations of the aggregated series negligible. Such a time series is represented by MA(1). Going through the derivations above, when there is only lag-1 correlation, it is seen that the adjustment factor is now closer to 1 . More specifically, the denominator can be replaced by the value 1 in factors (A4) and (A6).
Whether MA(1) is a reasonable description must be judged from data. Figure 5 illustrates the situation. With a time unit of 3 or 5 years we see a few significant lag- 2 correlations of magnitude 0.2 , but for longer time units the estimated lag $\geq 2$ autocorrelations look like what is expected for white noise. Even with a damping factor of 0.85 in an AR(1), we can conclude that the lag-2 correlation with a time unit of 8 years is reduced relative to the lag- 1 correlation by a factor of type $0.85^{8}=0.27$, and by one more such factor for lag-2, etc. Thus, if we use a time unit of 8 years and modify the test statistic variances by the factor in factor (A4), where $\rho$ is the lag- 1 correlation with this time unit, we should be on the safe side.

\section{Appendix B: The test statistics in the presence of a joint forcing}

Suppose we want to compare models which all include a particular underlying forcing that is not of current interest. One model has another, additional forcing that is of current interest, whereas another model is a reference model, corresponding to no effect of the additional forcing. In that situation, with its absence of a null model, the correlation test statistic $R$ cannot be judged on its own, but the correlation must be compared with that for the reference model. On the other hand, it will be shown that the $T$-based test statistic need not be adjusted at all. None of the forcing effects need be present in the true climate, but the tests discussed here are most likely of interest when the effect of the forcing of the reference model has already been detected in the observations or is assumed for physical reasons to affect the true climate. In Appendix B4, we extend the situation to discuss comparison of two alternative forcings of the same type, such as low- and high-amplitude solar forcing.

\section{B1 Model}

As in Appendix A1, suppose we have two climate models ${ }^{5}$, represented by simulation sequences $x$ and $x^{*}$, the latter having a role as reference, and an observation series for the same period, denoted $z$. The new feature is that we allow a "baseline" forcing present in both climate models, and probably also in the true temperature. This baseline forcing is not of current interest, but there is another, additional forcing applied in $x$ but not in $x^{*}$. As before, the hypothesis $H_{0}$ to be tested assumes this additional forcing has no effect.

Statistical model under $H_{0}$ : Climate model simulation sequences $\left\{x_{i}\right\}$ and $\left\{x_{i}^{*}\right\}$, true climate sequence $\left\{\tau_{i}\right\}$, and observation sequence $\left\{z_{i}\right\}$ are mutually related through the following model:

$x_{i}=\mu_{x}+\gamma_{i}+\delta_{i}$,

\footnotetext{
${ }^{5}$ See footnote in Appendix A1
} 
$x_{i}^{*}=\mu_{x}+\gamma_{i}+\delta_{i}^{*}$,

$\tau_{i}=\mu_{\tau}+\eta_{i}$

$z_{i}=\tau_{i}+\epsilon_{i}$

Here, the term $\gamma_{i}$, in common for $x$ and $x^{*}$, is the baseline forcing effect, regarded as of more or less random character. Thus, $x$ and $x^{*}$ differ only by separate random "noise" terms $\delta$ and $\delta^{*}$. All three terms $\gamma, \delta$ and $\delta^{*}$ are assumed mutually uncorrelated with time-constant variances (even for $\gamma$ when it is considered random, variance $\sigma_{\gamma}^{2}$ ). The baseline forcing may also be present in the true climate, and is even likely to be so. We therefore allow $\gamma_{i}$ to be correlated with the term $\eta_{i}$, with no need to be more specific.

In SUN12, the additional forcing of concern was represented by terms $\xi$ and $\alpha \xi$ in the expressions for $\tau$ and $x$, respectively, but here we need not be so explicit because they do not occur in the null model. We always know, of course, that such an additional forcing has been implemented in the climate model simulation represented by $x$ in the statistical model, but we want to investigate whether a response to this forcing is also seen in the observations. Our tests are designed to detect whether there is a strong enough such additional forcing effect jointly present in $x$ and $\tau$. If the test results lead to rejection of $H_{0}$, it indicates that there is an effect of the additional forcing in the true climate sequence $\tau$, because our test statistics are sensitive only to a joint effect in $x$ and $\tau$.

For simplicity we assume here that there is no autocorrelation in the $x$ sequences. However, such autocorrelation can be adjusted for as described in Appendix A, and we did so in our experiment. Generally, this is likely to be even more needed here than before, since the baseline forcing effect $\gamma$ is likely to contribute additional autocorrelation within the reference series.

Other terms representing unexplained variability (random fluctuations, noise) are $\eta_{i}$ and $\epsilon_{i}$, the $\eta_{i}$ term representing all variability in the true climate. However, we make no assumption about the true climate $\tau$ or the observed climate series $z$. In particular, it may contain more or less effect from the baseline forcing that was also behind the $\gamma$ term of the climate models. The reason we do not need assumptions is that we will consider statistics such as the difference between two $D^{2}$ values. The $D^{2}$ values themselves are typically reduced if we introduce a realistic forcing effect $\gamma_{i}$ in the models that is also present in the true climate. The difference between two such characteristics, however, will not be systematically changed. Technically, we regard the observed $z$ series as given and fixed, and the statistical analysis is conditional on this given series. The weights $w_{i} \geq 0$ and $\widetilde{w}_{i} \geq 0$ (see Sects. 5 and 8 in SUN12) will also be regarded as given and fixed. Concerning the definition of $\widetilde{w}_{i}$, note the footnote in Sect. 2 of the current study.

\section{B2 Correlation test statistic}

The test statistic denoted $R(x, z)$ in Sect. 8 of SUN12 differs only by a constant factor from

$\sum \widetilde{w}_{i}\left(x_{i}-\mu_{x}\right) z_{i}$.

Here the theoretical average $\mu_{x}$ will be replaced by the corresponding empirical average.

When a forcing is present in the reference model, we must expect that this forcing causes an underlying positive correlation with the observations on its own. For that reason we must bring in the reference $x^{*}$ and show that $x$, as compared with $x^{*}$, is more correlated with $z$. Therefore we use the difference $R(x, z)-R\left(x^{*}, z\right)$ instead of $R(x, z)$. The $\gamma$ term cancels, so given $z$, the difference consists of two mutually uncorrelated terms with variance twice that of the single term variance in Eq. (20) given in SUN12 as a function of $\sigma_{\delta}^{2}$. It only remains to remember what $\sigma_{\delta}^{2}$ stands for. This is the residual variance in the reference simulations $x^{*}$ after adjustment for the unknown forcing effect $\gamma$. But this variance is majorized by the total variance of $x^{*}$, obtained when we additionally include the variation of $\gamma_{i}, \operatorname{Var}\left(x_{i}^{*}\right)=\sigma_{\delta}^{2}+\operatorname{Var}\left(\gamma_{i}\right) \geq \sigma_{\delta}^{2}$ if $\gamma$ is regarded as random with a variance. When the $\gamma_{i}$ sequence is regarded as fixed, we instead state that $\sigma_{\delta}^{2}$ is overestimated by the total sample variance $s_{x}^{2}$ of the $x^{*}$ sequence. Thus we are on the safe side when using the sample variance of $x_{i}^{*}$, and in comparison with the results of SUN12 we need not bother about $\gamma_{i}$ but simply adjust the variance in their Eq. (20) by a factor 2 when $R(x, z)$ is replaced by $R(x, z)-R\left(x^{*}, z\right)$. Furthermore, in many cases the relative difference between $\sigma_{\delta}^{2}$ and $s_{x}^{2}$ will be small, so the majorization (upper bound) is not only on the safe side but also innocent.

\section{B3 $D^{2}$ difference test statistic}

The $D^{2}$ difference test statistic $T\left(x, x^{*}, z\right)=D_{\mathrm{W}}^{2}(x, z)-$ $D_{\mathrm{W}}^{2}\left(x^{*}, z\right)$ (see Eq. 1), can be expressed in the form

$$
\begin{aligned}
T\left(x, x^{*}, z\right) & =\overline{w\left(x-\mu_{x}\right)^{2}}-\overline{w\left(x^{*}-\mu_{x}\right)^{2}} \\
& -2 \overline{w\left(x-x^{*}\right)\left(z-\mu_{x}\right)},
\end{aligned}
$$

with overlines denoting averaging over time ( $n$ time steps). (Note: Eq. B2 is identical to Eq. A5.) Under $H_{0}$, saying that $x$ and its reference $x^{*}$ are equivalent, the first two terms have equal expected values and the third term with its $x-x^{*}$ will have an expected value of zero, so $T$ has an expected value of zero. This is true even when $x$ and $x^{*}$ have a term $\gamma$ in common. To form a test statistic we only additionally need the variance of $T$ under $H_{0}$. Because it simplifies the derivation, we will here regard the $\gamma$ term as random.

The first two terms of Eq. (B2) are mutually uncorrelated. Each of them is also uncorrelated with the third term, under an assumption of Gaussian noise $\delta$ and $\delta^{*}$ in $x$ and $x^{*}$, respectively (already made in SUN12). This is because the covariances will be proportional to the third-order central moments of $\delta$ (or $\delta^{*}$ ), which are zero because of the symmetry 
of the Gaussian distribution around its mean value (the only property needed, in fact). Note that $x-x^{*}=\delta-\delta^{*}$.

The third term of Eq. (B2) yields a variance that is formally the same as in SUN12. The only difference is (again, see the previous section) in the interpretation of the unknown $\sigma_{\delta}^{2}$. By using instead the sample variance of the reference $x^{*}$ sequence we get a useful upper bound.

The first two terms of Eq. (B2) have the same variance. In SUN12 this was given to be $2\left(\sigma_{\delta}^{2}\right)^{2}$, which was estimated by the sample variance of the reference model simulation. In the present case, when we consider $\gamma_{i}$ as random (and Gaussian and uncorrelated with $\delta_{i}$ ), we immediately get the same type of formula, but with $\operatorname{Var}(\gamma+\delta)=\sigma_{\gamma}^{2}+\sigma_{\delta}^{2}$ for $\sigma_{\delta}^{2}$. In practice, there is no difference, however, because the natural estimate of this variance is still the sample variance of the $x_{i}^{*}$ sequence.

We conclude that, also for the first two terms of Eq. (B2), we can use the formula of SUN12, with its $\sigma_{\delta}^{2}$ interpreted as the sample variance of the $x^{*}$ sequence. In other words, for the $T$-based test we can use the same calculation procedure as in SUN12, in particular their variance formulas in Eqs. (15) and (16), without bothering about $\gamma_{i}$, just pretending it does not exist. With the interpretation above, it does not matter what the $\gamma_{i}$ sequence is. We have an upper bound for the variance that will be close to the unknown true value unless the actual quadratic variation in the $\gamma_{i}$ sequence is a substantial part of the total variance. To adjust for autocorrelation in the reference model, the lag-1 correlation $\rho$ should be estimated from the sample $x^{*}$ sequence, and a variance adjustment should be made as in Appendix A.

\section{B4 Comparison of climate models with the same type of forcing}

Additionally, the result above can be used to compare two climate models of the same kind, but driven with alternative versions of the type of forcing of interest, to see whether one is significantly better than the other. We then test the hypothesis that the two models are equivalent, in the sense of having the same forcing and the same magnitude of the response to this forcing. Expressed in terms of the statistical model in Appendix B1 above, we test the hypothesis that the two simulation models have the same forcing effect term (the $\gamma$ term), and if their $D^{2}$ difference is statistically significant, we can conclude that one of the models fits better than the other. We do the same here as when we tested a forced model against an unforced control by forming a variance-normalized $D^{2}$ difference, although this test is now two-sided since none of the models is a reference. Thus we need the variance of the $D^{2}$ difference when both models have the same forcing effect, as in the previous section. A difference, though, is that the estimate of $\sigma_{\delta}^{2}$ is now naturally taken to be the average of the sample variances for the two models.
One additional comment is motivated here. If the two forcings of interest are truly different alternatives with somewhat different temporal evolution, then, clearly, none of the models is a reference. But if the two forcings are just differently scaled versions of the same basic data, thus differing only in their amplitude, then the one with the smaller amplitude could be regarded as a reference, at least for the correlation test.

In our experiment where we compared the E1 and E2 simulations, the situation is somewhere in between, as the solar forcings differ both in low-frequency amplitude and in temporal evolution. Because the different amplitude is of the largest interest, we decided to estimate $\sigma_{\delta}^{2}$ (and $\rho$ ) only from E1 (having the smaller solar forcing amplitude) but used a two-sided test for the result in Fig. 8. However, we also found that using an average of E1 and E2 parameter estimates hardly changed the results at all. A final note relates to Appendix A. When there is no hypothesis model free from forcing effects, we must expect and allow for more autocorrelation. This could motivate the use of longer time units than in the tests with a reference free from forcing effects. 
Acknowledgements. We thank the four anonymous referees for careful reading and constructive comments. We also thank Ekaterina Fetisova for fruitful discussion of details in our method. This research was funded by the Swedish Research Council (grants 90751501, B0334901 and C0592401; the first two given to the lead author and the third to Gudrun Brattström at the Department of Mathematics, Stockholm University).

Edited by: J. Luterbacher

\section{References}

Allen, M. R. and Tett, S. F. B.: Checking for model consistency in optimal fingerprinting, Clim. Dynam., 15, 419-434, 1999.

Ammann, C. M., Genton, M. G., and Li, B.: Technical Note: Correcting for signal attenuation from noisy proxy data in climate reconstructions, Clim. Past, 6, 273-279, doi:10.5194/cp-6-2732010, 2010.

Anchukaitis, K. J., D’Arrigo, R. D., Andreu-Hayles, L., Frank, D., Verstege, A., Curtis, A., Buckley, B. M., Jacoby, G. C., and Cook, E. R.: Tree-ring-reconstructed summer temperatures from northwestern North America during the last nine centuries, J. Climate, 26, 3001-3012, 2013.

Bard, E., Raisbeck, G., Yiou, F., and Jouzel, J.: Solar irradiance during the last 1200 years based on cosmogenic nuclides, Tellus B, 52, 985-992, 2000.

Box, G. E. P., Jenkins, G. M., and Reinsel, G. C.: Time series analysis: Forecasting and control, John Wiley \& Sons, Hoboken, N. J., 4th Edn., 2007.

Briffa, K. R., Jones, P. D., Bartholin, T. S., Eckstein, D., Schweingruber, F. H., Karlén, W., Zetterberg, P., and Eronen, M.: Fennoscandian summers from AD 500: temperature changes on short and long timescales, Clim. Dynam., 7, 111-119, 1992.

Briffa, K. R., Osborn, T. J., and Schweingruber, F. H.: Large-scale temperature inferences from tree rings: a review, Global Planet. Change, 40, 11-26, 2004.

Briffa, K. R., Shishov, V. V., Melvin, T. M., Vaganov, E. A., Grudd, H., Hantemirov, R. M., Eronen, M., and Naurzbaev, M. M.: Trends in recent temperature and radial tree growth spanning 2000 years across northwest Eurasia, Philos. Trans. R. Soc. London, Ser. B, 353, 2269-2282, 2008.

Briffa, K. R., Melvin, T. M., Osborn, T. J., Hantemirov, R. M., Kirdyanov, A. V., Mazepa, V. S., Shiyatov, S. G., and Esper, J.: Reassessing the evidence for tree-growth and inferred temperature change during the Common Era in Yamalia, northwest Siberia, Quaternary. Sci. Rev., 72, 83-107, 2013.

Brohan, P., Kennedy, J. J., Harris, I., Tett, S. F. B., and Jones, P. D.: Uncertainty estimates in regional and global observed temperature changes: a new data set from 1850, J. Geophys. Res. Atmos., 111, D12106, doi:10.1029/2005JD006548, 2006.

Büntgen, U., Frank, D. C., Nievergelt, D., and Esper, J.: Summer temperature variations in the European Alps, A.D. 755-2004, J. Climate, 19, 5606-5623, 2006.

Büntgen, U., Kyncl, T., Ginzler, C., Jacks, D. S., Esper, J., Tegel, W., and Heussner, K.-U.: Filling the eastern European gap in millennium-long temperature reconstructions, P. Natl. Acad. Sci. USA, 110, 1773-1778, 2013.
Charlton-Perez, A. J., Baldwin, M. P., Birner, T., Black, R. X., Butler, A. M., Calvo, N., Davis, N. A., Gerber, E. P., Gillett, N., Hardiman, S., Kim, J., Krüger, K., Lee, Y.-Y., Manzini, E., McDaniel, B. A., Polvani, L., Reichler, T., Shaw, T. A., Sigmond, M., Son, S.-W., Toohey, M., Wilcox, L., Yoden, S., Christiansen, B., Lott, F., Shindell, D., Yukimoto, S., and Watanabe, S.: On the lack of stratospheric dynamical variability in low-top versions of the CMIP5 models, J. Geophys. Res.-Atmos., 118, 2494-2505, doi:10.1002/jgrd.50125, 2013.

Cook, E. R., Briffa, K. R., Meko, D. M., Graybill, D. A., and Funkhouser, G.: The "segment length curse" in long tree-ring chronology development for palaeoclimatic studies, Holocene, 5, 229-237, 1995.

Cook, E. R., Buckley, M. M., D’Arrigo, R. D., and Peterson, M. J.: Warm-season temperatures since $1600 \mathrm{BC}$ reconstructed from Tasmanian tree rings and their relationship to large-scale sea surface temperature anomalies, Clim. Dynam., 16, 79-91, 2000.

Cook, E. R., Palmer, J. G., and D'Arrigo, R. D.: Evidence for a “Medieval Warm Period" in a 1100 year tree-ring reconstruction of past austral summer temperatures in New Zealand, Geophys. Res. Lett., 29, 12-1-12-4, 2002.

Cook, E. R., Buckley, B. M., Palmer, J. G., Fenwick, P., Peterson, M. J., Boswijk, G., and Fowler, A.: Millennia-long tree-ring records from Tasmania and New Zealand: a basis for modelling climate variability and forcing, past, present and future, J. Quaternary Sci., 21, 689-699, 2006.

Cook, E. R., Krusic, P. J., Anchukaitis, K. J., Buckley, B. M., Nakatsuka, T., and Sano, M.: Tree-ring reconstructed summer temperature anomalies for temperate East Asia since 800 C.E., Clim. Dynam., 41, 2957-2972, 2013.

D’Arrigo, R., Jacoby, G., Buckley, B., Sakulich, J., Frank, D., Wilson, R., Curtis, A., and Anchukaitis, K.: Tree growth and inferred temperature variability at the North American Arctic treeline, Global Planet. Change, 65, 71-82, 2009.

D’Arrigo, R. D., Wilson, R., and Jacoby, G.: On the long-term context for late twentieth century warming, J. Geophys. Res., 111, D03103, doi:10.1029/2005JD006352, 2006.

D'Arrigo, R. D., Wilson, R., and Anchukaitis, K. J.: Volcanic cooling signal in tree ring temperature records, J. Geophys. Res.Atmos., 118, 9000-9010, doi:10.1002/jgrd.50692, 2013.

Dorado Liñán, I., Büntgen, U., González-Rouco, F., Zorita, E., Montávez, J. P., Gómez-Navarro, J. J., Brunet, M., Heinrich, I., Helle, G., and Gutiérrez, E.: Estimating 750 years of temperature variations and uncertainties in the Pyrenees by tree-ring reconstructions and climate simulations, Clim. Past, 8, 919-933, doi:10.5194/cp-8-919-2012, 2012.

Esper, J., Frank, D. C., Timonen, M., Zorita, E., Wilson, R. J. S., Luterbacher, J., Holzkamper, S., Fischer, N., Wagner, S., Nievergelt, D., Verstege, A., and Büntgen, U.: Orbital forcing of treering data, Nature Climate Change, 2, 862-866, 2012.

Esper, J., Schneider, L., Krusic, P. J., Luterbacher, J., Büntgen, U., Timonen, M., Sirocko, F., and Zorita, E.: European summer temperature response to annually dated volcanic eruptions over the past nine centuries, B. Volcanol., 75, 1-14, 2013.

Fernández-Donado, L., González-Rouco, J. F., Raible, C. C., Ammann, C. M., Barriopedro, D., García-Bustamante, E., Jungclaus, J. H., Lorenz, S. J., Luterbacher, J., Phipps, S. J., Servonnat, J., Swingedouw, D., Tett, S. F. B., Wagner, S., Yiou, P., and Zorita, E.: Large-scale temperature response to external forcing in simu- 
lations and reconstructions of the last millennium, Clim. Past, 9, 393-421, doi:10.5194/cp-9-393-2013, 2013.

Franke, J., Frank, D., Raible, C. C., Esper, J., and Brönnimann, S.: Spectral biases in tree-ring climate proxies, Nature Climate Change, 3, 360-364, 2013.

Fritts, H. C.: Tree Rings and Climate, Academic Press, London, 1976.

Goosse, H., Crespin, E., Dubinkina, S., Loutre, M.-F., Mann, M. E., Renssen, H., Sallaz-Damaz, Y., and Shindell, D.: The role of forcing and internal dynamics in explaining the "Medieval Climate Anomaly", Clim. Dynam., 39, 2847-2866, 2012.

Gray, L. J., Beer, J., Geller, M., Haigh, J. D., Lockwood, M., Matthes, K., Cubasch, U., Fleitmann, D., Harrison, G., Hood, L., Luterbacher, J., Meehl, G. A., Shindell, D., van Geel, B., and White, W.: Solar influences on climate, Rev. Geophys., 48, RG4001, doi:10.1029/2009RG000282, 2010.

Gunnarson, B. E., Linderholm, H. W., and Moberg, A.: Improving a tree-ring reconstruction from west-central Scandinavia: 900 years of warm-season temperatures, Clim. Dynam., 36, 97-108, 2011.

Hansen, J., Ruedy, R., Sato, M., and Lo, K.: Global surface temperature change, Rev. Geophys., 48, RG4004, doi:10.1029/2010RG000345, 2010.

Hasselmann, K.: Stochastic climate models. Part I. Theory, Tellus, 28, 473-485, 1976.

Hegerl, G. C., Crowley, T. J., Hyde, W. T., and Frame, D. J.: Climate sensitivity constrained by temperature reconstructions over the past seven centuries, Nature, 440, 1029-1032, 2006.

Hegerl, G. C., Crowley, T. J., Allen, M., Hyde, W. T., Pollack, H. N., Smerdon, J., and Zorita, E.: Detection of human influence on a new, validated 1500-year temperature reconstruction, J. Climate, 20, 650-666, 2007.

Hegerl, G. C., Luterbacher, J., González-Rouco, F., Tett, S. F. B., Crowley, T., and Xoplaki, E.: Influence of human and natural forcing on European seasonal temperatures, Nat. Geosci., 4, 99103, 2011.

Hind, A. and Moberg, A.: Past millennial solar forcing magnitude. A statistical hemispheric-scale climate model versus proxy data comparison, Clim. Dynam., 41, 2527-2537, 2013.

Hind, A., Moberg, A., and Sundberg, R.: Statistical framework for evaluation of climate model simulations by use of climate proxy data from the last millennium - Part 2: A pseudo-proxy study addressing the amplitude of solar forcing, Clim. Past, 8, 13551365, doi:10.5194/cp-8-1355-2012, 2012.

Hughes, M. K.: Dendrochronology in climatology - the state of the art, Dendrochronologia, 20, 95-116, 2002.

Hughes, M. K., Swetnam, T. W., and Diaz, H. F.: Dendroclimatology, in: Progress and Prospects, Springer, Dordrecht, Heidelberg, London, New York, 2011.

Jones, P. D., Briffa, K. R., Osborn, T. J., Lough, J. M., van Ommen, T. D., Vinther, B. M., Luterbacher, J., Wahl, E. R., Zwiers, F. W., Mann, M. E., Schmidt, G. A., Ammann, C. M., Buckley, B. M., Cobb, K. M., Esper, J., Goosse, H., Graham, N., Jansen, E., Kiefer, T., Kull, C., Küttel, M., Mosley-Thompson, E., Overpeck, J. T., Riedwyl, N., Schulz, M., Tudhope, A. W., Villalba, R., Wanner, H., Wolff, E., and Xoplaki, E.: High-resolution palaeoclimatology of the last millennium: a review of current status and future prospects, Holocene, 19, 3-49, 2009.
Jones, P. D., Melvin, T. M., Harpham, C., Grudd, H., and Helama, S.: Cool north European summers and possible links to explosive volcanic eruptions, J. Geophys. Res.-Atmos., 118, 6259-6265, 2013.

Jungclaus, J. H., Lorenz, S. J., Timmreck, C., Reick, C. H., Brovkin, V., Six, K., Segschneider, J., Giorgetta, M. A., Crowley, T. J., Pongratz, J., Krivova, N. A., Vieira, L. E., Solanki, S. K., Klocke, D., Botzet, M., Esch, M., Gayler, V., Haak, H., Raddatz, T. J., Roeckner, E., Schnur, R., Widmann, H., Claussen, M., Stevens, B., and Marotzke, J.: Climate and carbon-cycle variability over the last millennium, Clim. Past, 6, 723-737, doi:10.5194/cp-6723-2010, 2010.

Krivova, N. A., Balmaceda, L., and Solanki, S. K.: Reconstruction of solar total irradiance since 1700 from the surface magnetic flux, Astron. Astrophys., 467, 335-346, 2007.

Kutzbach, L., Thees, B., and Wilmking, M.: Identification of linear relationships from noisy data using errors-in-variables models relevance for reconstruction of past climate from tree-ring and other proxy information, Climatic Change, 105, 155-177, 2011.

Landrum, L., Otto-Bliesner, B. L., Wahl, E. R., Conley, A., Lawrence, P. J., Rosenbloom, N., and Teng, H.: Last millennium climate and its variability in CCSM4, J. Climate, 26, 1085-1111, 2012.

Lockwood, M.: Shining a light on solar impacts, Nature Climate Change, 1, 98-99, 2011.

Luckman, B. and Wilson, R.: Summer temperatures in the Canadian Rockies during the last millennium: a revised record, Clim. Dynam., 24, 131-144, 2005.

Masson, D. and Knutti, R.: Spatial-scale dependence of climate model performance in the CMIP3 ensemble, J. Climate, 24, 2680-2692, 2011.

Masson-Delmotte, V., Schulz, M., Abe-Ouchi, A., Beer, J., Ganopolski, A., González Rouco, J. F., Jansen, E., Lambeck, K., Luterbacher, J., Naish, T., Osborn, T., Otto-Bliesner, B., Quinn, T., Ramesh, R., Rojas, M., Shao, X., and Timmermann, A.: Information from Paleoclimate Archives, in: Climate Change 2013: The Physical Science Basis. Contribution of Working Group I to the Fifth Assessment Report of the Intergovernmental Panel on Climate Change, edited by: Stocker, T. F., Qin, D., Plattner, G.K., Tignor, M., Allen, S. K., Boschung, J., Nauels, A., Xia, Y., Bex, V., and Midgley, P. M., Cambridge University Press, Cambridge, UK and New York, NY, USA, 2013.

Matlab: Statistics Toolbox User's Guide R2008a, The MathWorks, Inc., Natick, MA, USA, 2008.

Melvin, T. M. and Briffa, K. R.: A "signal-free" approach to dendroclimatic standardisation, Dendrochronologia, 26, 71-86, 2008.

Melvin, T. M. and Briffa, K. R.: CRUST: Software for the implementation of regional chronology standardisation: Part 1. Signalfree RCS, Dendrochronologia, 32, 7-20, 2014.

Melvin, T. M., Grudd, H., and Briffa, K. R.: Potential bias in "updating" tree-ring chronologies using regional curve standardisation: re-processing 1500 years of Torneträsk density and ringwidth data, Holocene, 23, 364-373, 2013.

Moberg, A. and Brattström, G.: Prediction intervals for climate reconstructions with autocorrelated noise - An analysis of ordinary least squares and measurement error methods, Palaeogeogr. Palaeocl., 308, 313-329, 2011.

Morice, C. P., Kennedy, J. J., Rayner, N. A., and Jones, P. D.: Quantifying uncertainties in global and regional tempera- 
ture change using an ensemble of observational estimates: the HadCRUT4 data set, J. Geophys. Res., 117, D08101, doi:10.1029/2011JD017187, 2012.

Osborn, T. J., Briffa, K. R., and Jones, P. D.: Adjusting variance for sample size in tree-ring chronologies and other regional mean timeseries, Dendrochronologia, 15, 89-99, 1997.

PAGES2k Network: Continental-scale temperature variability during the past two millennia, Nat. Geosci., 6, 339-346, 2013.

Phipps, S. J., McGregor, H. V., Gergis, J., Gallant, A. J. E., Neukom, R., Stevenson, S., Ackerley, D., Brown, J. R., Fischer, M. J., and van Ommen, T. D.: Paleoclimate data-model comparison and the role of climate forcings over the past 1500 years, J. Climate, 26, 6915-6936, 2013.

Schmidt, G. A.: Enhancing the relevance of palaeoclimate model/data comparisons for assessments of future climate change, J. Quaternary Sci., 25, 79-87, 2010.

Schmidt, G. A., Jungclaus, J. H., Ammann, C. M., Bard, E., Braconnot, P., Crowley, T. J., Delaygue, G., Joos, F., Krivova, N. A., Muscheler, R., Otto-Bliesner, B. L., Pongratz, J., Shindell, D. T., Solanki, S. K., Steinhilber, F., and Vieira, L. E. A.: Climate forcing reconstructions for use in PMIP simulations of the last millennium (v1.0), Geosci. Model Dev., 4, 33-45, doi:10.5194/gmd4-33-2011, 2011.

Schmidt, G. A., Jungclaus, J. H., Ammann, C. M., Bard, E., Braconnot, P., Crowley, T. J., Delaygue, G., Joos, F., Krivova, N. A., Muscheler, R., Otto-Bliesner, B. L., Pongratz, J., Shindell, D. T., Solanki, S. K., Steinhilber, F., and Vieira, L. E. A.: Climate forcing reconstructions for use in PMIP simulations of the Last Millennium (v1.1), Geosci. Model Dev., 5, 185-191, doi:10.5194/gmd-5-185-2012, 2012.

Schurer, A., Hegerl, G., Mann, M., Tett, S., and Phipps, S.: Separating forced from chaotic climate variability over the past millennium, J. Climate, 26, 6954-6973, 2013.

Schurer, A., Tett, S. F. B, and Hegerl, G. C.: Small influence of solar variability over the past millennium, Nat. Geosci., 7, 104-108, 2014.

Servonnat, J., Yiou, P., Khodri, M., Swingedouw, D., and Denvil, S.: Influence of solar variability, $\mathrm{CO}_{2}$ and orbital forcing between 1000 and 1850 AD in the IPSLCM4 model, Clim. Past, 6, 445460, doi:10.5194/cp-6-445-2010, 2010.
Smith, T. M., Reynolds, R. W., Peterson, T. C., and Lawrimore, J.: Improvement to NOAA's historical merged land-ocean surface temperature analysis (1880-2006), J. Climate, 21, 2283-2296, 2008.

St. George, S. and Ault, T.: The imprint of climate within Northern Hemisphere trees, Quaternary. Sci. Rev., 89, 1-4, 2014.

Sueyoshi, T., Ohgaito, R., Yamamoto, A., Chikamoto, M. O., Hajima, T., Okajima, H., Yoshimori, M., Abe, M., O'ishi, R., Saito, F., Watanabe, S., Kawamiya, M., and Abe-Ouchi, A.: Set-up of the PMIP3 paleoclimate experiments conducted using an Earth system model, MIROC-ESM, Geosci. Model Dev., 6, 819-836, doi:10.5194/gmd-6-819-2013, 2013.

Sundberg, R., Moberg, A., and Hind, A.: Statistical framework for evaluation of climate model simulations by use of climate proxy data from the last millennium - Part 1: Theory, Clim. Past, 8, 1339-1353, doi:10.5194/cp-8-1339-2012, 2012.

Trouet, V. and van Oldenborgh, G. J.: KNMI Climate Explorer: a web-based research tool for high-resolution paleoclimatology, Tree Ring Research, 69, 3-13, doi:10.3959/1536-1098-69.1.3, 2013.

Vyushin, D. I., Kushner, P. J., and Zwiers, F.: Modeling and understanding persistence of climate variability, J. Geophys. Res., 117, D21106, doi:10.1029/2012JD018240, 2012.

Wanner, H., Beer, J., Bütikofer, J., Crowley, T., Cubasch, U., Flückiger, J., Goosse, H., Grosjean, M., Joos, F., Kaplan, J., Küttel, M., Müller, S., Prentice, I., Solomina, O., Stocker, T., Tarasov, P., Wagner, M., and Widmann, M.: Mid- to Late Holocene climate change: an overview, Quaternary Sci. Rev., 27, 1791-1828, 2008.

Widmann, M., Goosse, H., van der Schrier, G., Schnur, R., and Barkmeijer, J.: Using data assimilation to study extratropical Northern Hemisphere climate over the last millennium, Clim. Past, 6, 627-644, doi:10.5194/cp-6-627-2010, 2010.

Wilson, R., Wiles, G., D'Arrigo, R., and Zweck, C.: Cycles and shifts: 1300 years of multi-decadal temperature variability in the Gulf of Alaska, Clim. Dynam., 28, 425-440, 2007.

Wilson, R. J. S.: Eurasian Regional Composite Chronologies, Research report prepared for Rosanne D'Arrigo and Gordon Jacoby of the Lamont Doherty Earth Observatory (LDEO) Tree-Ring Laboratory, Columbia University, New York, 31 pp., 2004. 